\title{
INFRARED SPECTRA AND PHOTOMETRY OF COMPLETE SAMPLES OF PALOMAR-GREEN AND TWO MICRON ALL SKY SURVEY QUASARS
}

\author{
Yong SHI ${ }^{1,2}$, G. H. RIEKE ${ }^{3}$, P. M. OGLE ${ }^{4}$, K. Y. L. SU ${ }^{3}$, AND Z. BALOG ${ }^{5}$ \\ ${ }^{1}$ School of Astronomy and Space Science, Nanjing University, Nanjing 210093, China; yshipku@ gmail.com \\ ${ }^{2}$ Key Laboratory of Modern Astronomy and Astrophysics (Nanjing University), Ministry of Education, Nanjing 210093, China \\ ${ }^{3}$ Department of Astronomy And Steward Observatory, University of Arizona, 933 North Cherry Avenue, Tucson, AZ 85721, USA \\ ${ }^{4}$ Infrared Processing and Analysis Center, California Institute of Technology, 1200 East California Boulevard, Pasadena, CA 91125, USA \\ 5 Max-Planck-Institut für Astronomie, Königstuhl 17, D-69117 Heidelberg, Germany \\ Received 2014 May 13; accepted 2014 August 7; published 2014 September 18
}

\begin{abstract}
As a step toward a comprehensive overview of the infrared (IR) diagnostics of the central engines and host galaxies of quasars at low redshift, we present Spitzer Space Telescope spectroscopic (5-40 $\mu \mathrm{m})$ and photometric (24, 70 , and $160 \mu \mathrm{m}$ ) measurements of all Palomar-Green (PG) quasars at $z<0.5$ and Two Micron All Sky Survey (2MASS) quasars at $z<0.3$. We supplement these data with Herschel measurements at $160 \mu \mathrm{m}$. The sample is composed of 87 optically selected PG quasars and 52 near-IR-selected 2MASS quasars. Here we present the data, measure the prominent spectral features, and separate emission due to star formation from that emitted by the dusty circumnuclear torus. We find that the mid-IR $(5-30 \mu \mathrm{m})$ spectral shape for the torus is largely independent of quasar IR luminosity with scatter in the spectral energy distribution (SED) shape of $\lesssim 0.2$ dex. Except for the silicate features, no large difference is observed between PG (unobscured-silicate emission) and 2MASS (obscured-silicate absorption) quasars. Only mild silicate features are observed in both cases. When in emission, the peak wavelength of the silicate feature tends to be longer than $9.7 \mu \mathrm{m}$, possibly indicating effects on grain properties near the active galactic nucleus. The IR color is shown to correlate with the equivalent width of the aromatic features, indicating that the slope of the quasar mid- to far-IR SED is to first order driven by the fraction of radiation from star formation in the IR bands.
\end{abstract}

Key words: galaxies: active - galaxies: starburst - infrared: galaxies

Online-only material: color figures, extended figure, machine-readable table

\section{INTRODUCTION}

Active galactic nuclei (AGNs), signposts for accretion onto super-massive black holes (SMBHs), are now understood to be a critical phase of massive galaxy formation. In the local universe all individual massive galaxies are found to host SMBHs at their centers, with the SMBH masses tightly correlated with galaxy properties (Kormendy \& Richstone 1995; Magorrian et al. 1998; Gebhardt et al. 2000; Ferrarese \& Merritt 2000). Probing the properties of material located at different radii surrounding SMBHs should offer important clues to how accretion by the SMBHs influences, or is influenced by, the evolution of their host galaxies to maintain this correlation. It may also solve puzzles such as how the angular momentum of material at kiloparsec radii is removed to feed central engines.

It is believed that there is a geometrically thick structure, termed the dusty torus, outside the accretion disk in AGNs. The dusty torus may physically bridge from the inner accretion disk to the outer host galaxy, and likely plays a crucial role in funneling the material into SMBHs. Significant progress has been made in probing the torus, starting from the earliest suggestions of its existence in the 1980s to direct imaging of it in a few nearby AGNs (Jaffe et al. 2004). The dusty torus is thought to explain the large apparent diversity of AGN phenomena. For example, in the AGN unified model (Antonucci 1993), orientation-dependent obscuration in the torus determines whether the broad emission lines emerge in the UV/optical spectra and thus whether an AGN is classified as type 1 or type 2 .
The dust in the torus is heated by UV/optical radiation from the accretion disk to high temperatures $(\sim 100-1000 \mathrm{~K})$ to emit dominantly in the near- to mid-infrared (IR) spectral ranges. The emission by the torus dust is dominated by a continuum that is featureless, except for the broad silicate features at around 9.7 and $18 \mu \mathrm{m}$ either in emission or absorption. Its output is distinct from the radiation of star-forming regions: for them, the aromatic features dominate at wavelengths shorter than $15 \mu \mathrm{m}$, while at longer wavelengths there is a strong continuum due to warm and cold $(<60 \mathrm{~K})$ dust (Smith et al. 2007; Stierwalt et al. 2013). In luminous AGNs, i.e., quasars such as those studied here, the torus nearly always dominates the integrated mid-IR emission so that the observations can be compared directly to predictions of torus models.

The silicate features are important diagnostics to constrain the structure of the dusty torus (Siebenmorgen et al. 2005; Hao et al. 2005; Sturm et al. 2005, 2006; Shi et al. 2006). They can be directly compared to predictions of dusty torus models, probing the internal torus structures (Fritz et al. 2006; Hönig et al. 2006; Nenkova et al. 2008; Schartmann et al. 2008; Stalevski et al. 2012; Feltre et al. 2012). For example, Feltre et al. (2012) demonstrate how to discriminate two broad types of dusty tori, i.e., smooth versus clumpy, through the behavior of the silicate features and of the overall mid-IR spectral energy distributions (SEDs). The IR emission lines also have the advantage of probing narrow emission line regions without significant extinction (e.g., Diamond-Stanic et al. 2009).

The IR data are also powerful in probing the host galaxy properties, e.g., the star formation rate (SFR). While commonly 
used SFR tracers, such as UV radiation, hydrogen recombination lines, and forbidden lines are contaminated severely by the nuclear radiation, the mid-IR aromatic features and far-IR photometry are two relatively uncontaminated tracers of star formation (SF) in quasars (e.g., Shi et al. 2007; Netzer et al. 2007; Lutz et al. 2008; Hernán-Caballero et al. 2009; Hiner et al. 2009; Rosario et al. 2013).

Studies of IR features are enabled by the Spitzer Space Telescope with a photometric wavelength coverage from 3.6 to $160 \mu \mathrm{m}$ and low-resolution spectroscopy from 5 to $40 \mu \mathrm{m}$. To exploit the potential of the IR data, we carried out a program with Spitzer in the last cryogenic cycle to complete spectroscopic $(5-40 \mu \mathrm{m})$ and photometric $(24,70$, and $160 \mu \mathrm{m})$ observations of two samples of luminous AGNs at low redshift, namely the unobscured optically selected Palomar-Green (PG) quasars (Schmidt \& Green 1983; Boroson \& Green 1992) and obscured near-IR selected Two Micron All Sky Survey (2MASS) quasars (Cutri et al. 2001; Smith et al. 2002). By focusing on quasars, we will probe the phase where SMBHs grow most rapidly and where their output stands out most clearly from that of their host galaxies.

In this paper we present the observations and extract measurements of aromatic and silicate features and continuum luminosities for these two samples. We use this information to determine the SFRs of the host galaxies and to show that the values from the aromatic bands and far-IR emission are consistent. We present the sample, observations, and data reductions in Section 2. The spectral decomposition is detailed in Section 3. The results are presented in Section 4. Our conclusions are presented Section 5. Throughout this paper, we assume $H_{0}=70 \mathrm{~km} \mathrm{~s}^{-1} \mathrm{Mpc}^{-1}, \Omega_{0}=0.3$, and $\Omega_{\Lambda}=0.7$.

\section{SAMPLES, OBSERVATIONS, AND DATA REDUCTION}

\subsection{Samples}

For this study we included all 87 objects of the PG sample (Schmidt \& Green 1983; Boroson \& Green 1992) at $z<0.5$ and all 52 objects of the 2MASS sample (Cutri et al. 2001; Smith et al. 2002) at $z<0.3$, as listed in Table 1 . The PG quasars are defined by an average limiting $B$-band magnitude of 16.16 , blue $U-B$ color $(<-0.44)$, and dominant starlike appearance. All these objects show broad emission lines, and thus are classified as type 1 quasars. Due to the large photographic magnitude errors and the simple color selection, the PG sample is incomplete (e.g., Goldschmidt et al. 1992; Jester et al. 2005), but the incompleteness is independent of the optical magnitude and color (Jester et al. 2005), indicating that the PG sample is still representative of bright optically selected quasars. Compared to PG quasars, the 2MASS quasars represent a redder population with $J-K_{s}>2$ (compared with a typical value of $J-K_{s} \sim 1.5$ for PG quasars), but have similar $K_{s}$-band luminosities $\left(M_{K_{s}}<-23\right.$; Smith et al. 2002). Unlike PG quasars, the 2MASS sample includes objects with narrow, intermediate and broad emission lines. The 2MASS sample is increasingly incomplete at $K_{s}>13$ (Cutri et al. 2001).

\subsection{Observations and Data Reduction}

We carried out 25.1 hr of Spitzer observations (PID: 50196; PI: G. Rieke) to complete the Infrared Spectrograph (IRS) spectroscopic and MIPS photometric data for the PG/2MASS sample. The program included all objects that did not have archived data from previous cycles. As a result, there are lowresolution IR spectra $(5-40 \mu \mathrm{m})$ and MIPS photometry at 24,
70 , and $160 \mu \mathrm{m}$ for the entire sample. ${ }^{6}$ All the broad-band photometry is listed in Table 2, while the IRS data are available in Table 3.

The spectra were obtained using the standard IRS staring mode. To reduce them, we drew from the archive the basic calibrated data (BCD) as products of Spitzer Science Center data reduction pipeline version $\mathrm{S} 18.7$, which provided cosmic ray removal, replacement of saturated pixels, droop correction, subtraction of darks, linearization correction, and stray light and flat field correction. The post-pipeline processing of the BCD was based on IRSCLEAN ${ }^{7}$ and SPICE. ${ }^{8}$ IRSCLEAN was used to create bad pixel masks for the BCD image. The sky background for each module within a given order was then subtracted using the image obtained with the same module in a different order. For observations with only one order, the image at one slit position was used as sky background for the image at another slit position. SPICE was employed to extract the spectra from these background-subtracted images. We used the optimal extraction mode to increase the ratio of signal to noise, since our targets are point sources. The mismatch in the signal between short-low $(5-14 \mu \mathrm{m})$ and longlow $(14-40 \mu \mathrm{m})$ modules was removed by scaling the short-low spectrum to the long-low one. The whole spectrum was further scaled to the MIPS $24 \mu \mathrm{m}$ photometry by comparing to synthetic photometry of the spectra. The flux difference between the shortlow and long-low, and the difference between the MIPS $24 \mu \mathrm{m}$ photometry and IRS synthetic flux at this wavelength, could be caused by the errors in the flux calibration of the MIPS and IRS instruments, and the pointing errors with short-low and longlow, not necessarily by extended emission outside the IRS slit. As listed in Table 2, the scaling factor from short-low to longlow has a median value of 1.0 and a standard deviation of 0.4 , and the factor from long-low synthetic $24 \mu \mathrm{m}$ to MIPS $24 \mu \mathrm{m}$ flux has a median value of 1.08 and a standard deviation of 0.18 . The almost unity conversion factor with scatter comparable to the flux calibration uncertainties indicates that missed extended emission is not important for the IRS observations. In addition, visual checks of MIPS $24 \mu \mathrm{m}$ images do not find any source with significant extended emission.

MIPS observations were made with the small field photometry mode. The data reduction and photometry measurements were carried out with Data Analysis tool v3.1 and redMIPS v1.1 (Gordon et al. 2005) by the MIPS Instrument team. For the $160 \mu \mathrm{m}$ photometry, we have visually checked individual images and removed those where the photometry is affected by close companions or structure in the sky (e.g., IR cirrus). In total $98 \%, 91 \%$, and $32 \%$ of the sample have MIPS detections above $3 \sigma$ at 24,70 , and $160 \mu \mathrm{m}$, respectively.

To increase the fraction of objects with $160 \mu \mathrm{m}$ detections, we searched for measurements in the Herschel Space Telescope data archive. All the PG quasars have been observed with Herschel (PI: L. Ho) but no archived data were found for the 2MASS

\footnotetext{
6 The previous IRS spectroscopic programs were by J. Houck (PID: 4), G. Rieke (PID: 36), F. Low (PID: 40), M. Werner (PID: 61), S. Gallagher (PID: 148), R. Siebenmorgen (PID: 193), D. Lutz (PID: 323), S. Veilleux (PID: 485), A. Stockton (PID: 14067), A. Wehrle (PID: 14991), and particularly by P. Ogle (PID: 11451). The previous MIPS programs were led by F. Low (PID: 49), G. Rieke (PID: 82, 30306, 40053, 50507), M. Werner (PID: 86), M. Bondi (PID: 3327), Z. Shang (PID: 20084), A. Marscher (PID: 20496), A. Wehrle (PID: 30785), and G. Fazio (PID: 30860).

7 http://irsa.ipac.caltech.edu/data/SPITZER/docs/dataanalysistools/tools/ irsclean/

8 http://irsa.ipac.caltech.edu/data/SPITZER/docs/dataanalysistools/tools/ spice/
} 
Table 1

Physical Properties of Our Sample

\begin{tabular}{|c|c|c|c|c|c|c|}
\hline $\begin{array}{l}\text { Number } \\
\text { (1) }\end{array}$ & $\begin{array}{l}\text { Name } \\
(2)\end{array}$ & $\begin{array}{c}\text { Position(J2000) } \\
\text { (3) }\end{array}$ & $\begin{array}{c}z \\
(4)\end{array}$ & $\begin{array}{l}m_{B} \\
(5)\end{array}$ & $\begin{array}{l}m_{K} \\
(6)\end{array}$ & $\begin{array}{l}\text { Type } \\
\text { (7) }\end{array}$ \\
\hline 1 & PG0003+158 & $00^{\mathrm{h}} 05^{\mathrm{m}} 59^{\mathrm{s}} \cdot 24+16^{\mathrm{d}} 09^{\mathrm{m}} 49^{\mathrm{s}} \cdot 0$ & 0.450 & 15.960 & & 1.0 \\
\hline 2 & PG0003+199 & $00^{\mathrm{h}} 06^{\mathrm{m}} 19 \mathrm{~s} .52+20^{\mathrm{d}} 12^{\mathrm{m}} 10^{\mathrm{s}} .5$ & 0.025 & 13.750 & & 1.0 \\
\hline 3 & PG0007+106 & $00^{\mathrm{h}} 10^{\mathrm{m}} 31^{\mathrm{s}} .01+10^{\mathrm{d}} 58^{\mathrm{m}} 29^{\mathrm{s}} .5$ & 0.089 & 16.110 & & 1.0 \\
\hline 4 & PG0026+129 & $00^{\mathrm{h}} 29^{\mathrm{m}} 13^{\mathrm{s}} \cdot 60+13^{\mathrm{d}} 16^{\mathrm{m}} 03^{\mathrm{s}} \cdot 0$ & 0.142 & 14.950 & & 1.0 \\
\hline 5 & PG0043+039 & $00^{\mathrm{h}} 45^{\mathrm{m}} 47^{\mathrm{s}} \cdot 27+04^{\mathrm{d}} 10^{\mathrm{m}} 24^{\mathrm{s}} .4$ & 0.384 & 15.880 & & 1.0 \\
\hline 6 & PG0049+171 & $00^{\mathrm{h}} 51^{\mathrm{m}} 54^{\mathrm{s}} .80+17^{\mathrm{d}} 25^{\mathrm{m}} 58^{\mathrm{s}} .4$ & 0.064 & 15.880 & & 1.0 \\
\hline 7 & PG0050+124 & $00^{\mathrm{h}} 53^{\mathrm{m}} 34^{\mathrm{s}} .94+12^{\mathrm{d}} 41^{\mathrm{m}} 36^{\mathrm{s}} .2$ & 0.061 & 14.390 & & 1.0 \\
\hline 8 & PG0052+251 & $00^{\mathrm{h}} 54^{\mathrm{m}} 52^{\mathrm{s}} .10+25^{\mathrm{d}} 25^{\mathrm{m}} 38.0$ & 0.155 & 15.420 & & 1.0 \\
\hline 9 & PG0157+001 & $01^{\mathrm{h}} 59^{\mathrm{m}} 50^{\mathrm{s}} .21+00^{\mathrm{d}} 23^{\mathrm{m}} 40^{\mathrm{s}} 6$ & 0.164 & 15.200 & & 1.0 \\
\hline 10 & PG0804+761 & $08^{\mathrm{h}} 10^{\mathrm{m}} 58^{\mathrm{s}} \cdot 60+76^{\mathrm{d}} 02^{\mathrm{m}} 42^{\mathrm{s}} \cdot 0$ & 0.100 & 15.150 & & 1.0 \\
\hline 11 & PG0838+770 & $08^{\mathrm{h}} 44^{\mathrm{m}} 45^{\mathrm{s}} \cdot 26+76^{\mathrm{d}} 53^{\mathrm{m}} 09^{\mathrm{s}} .5$ & 0.131 & 16.300 & & 1.0 \\
\hline 12 & PG0844+349 & $08^{\mathrm{h}} 47^{\mathrm{m}} 42^{\mathrm{s}} .47+34^{\mathrm{d}} 45^{\mathrm{m}} 04^{\mathrm{s}} .4$ & 0.064 & 14.000 & & 1.0 \\
\hline 13 & PG0921+525 & $09^{\mathrm{h}} 25^{\mathrm{m}} 12^{\mathrm{s}} .87+52^{\mathrm{d}} 17^{\mathrm{m}} 10^{\mathrm{s}} .5$ & 0.035 & 15.620 & & 1.0 \\
\hline 14 & PG0923+201 & $09^{\mathrm{h}} 25^{\mathrm{m}} 54^{\mathrm{s}} .72+19^{\mathrm{d}} 54^{\mathrm{m}} 05^{\mathrm{s}} .1$ & 0.190 & 16.040 & & 1.0 \\
\hline 15 & PG0923+129 & $09^{\mathrm{h}} 26^{\mathrm{m}} 03^{\mathrm{s}} \cdot 29+12^{\mathrm{d}} 44^{\mathrm{m}} 03^{\mathrm{s}} \cdot 6$ & 0.029 & 14.930 & & 1.0 \\
\hline 16 & PG0934+013 & $09^{\mathrm{h}} 37^{\mathrm{m}} 01^{\mathrm{s}} .03+01^{\mathrm{d}} 05^{\mathrm{m}} 43^{\mathrm{s}} .5$ & 0.050 & 16.290 & & 1.0 \\
\hline 17 & PG0947+396 & $09^{\mathrm{h}} 50^{\mathrm{m}} 48^{\mathrm{s}} 39+39^{\mathrm{d}} 26^{\mathrm{m}} 50^{\mathrm{s}} .5$ & 0.206 & 16.400 & & 1.0 \\
\hline 18 & PG0953+414 & $09^{\mathrm{h}} 56^{\mathrm{m}} 52^{\mathrm{s}} \cdot 39+41^{\mathrm{d}} 15^{\mathrm{m}} 22^{\mathrm{s}} \cdot 3$ & 0.239 & 15.050 & & 1.0 \\
\hline 19 & PG1001+054 & $10^{\mathrm{h}} 04^{\mathrm{m}} 20^{\mathrm{s}} \cdot 14+05^{\mathrm{d}} 13^{\mathrm{m}} 00^{\mathrm{s}} .5$ & 0.161 & 16.130 & & 1.0 \\
\hline 20 & PG1004+130 & $10^{\mathrm{h}} 07^{\mathrm{m}} 26^{\mathrm{s}} \cdot 10+12^{\mathrm{d}} 48^{\mathrm{m}} 56^{\mathrm{s}} \cdot 2$ & 0.240 & 15.930 & & 1.0 \\
\hline 21 & PG1011-040 & $10^{\mathrm{h}} 14^{\mathrm{m}} 20^{\mathrm{s}} .69-04^{\mathrm{d}} 18^{\mathrm{m}} 40^{\mathrm{s}} .5$ & 0.058 & 15.490 & & 1.0 \\
\hline 22 & PG1012+008 & $10^{\mathrm{h}} 14^{\mathrm{m}} 54^{\mathrm{s}} \cdot 90+00^{\mathrm{d}} 33^{\mathrm{m}} 37^{\mathrm{s}} \cdot 4$ & 0.185 & 15.890 & & 1.0 \\
\hline 23 & PG1022+519 & $10^{\mathrm{h}} 25^{\mathrm{m}} 31^{\mathrm{s}} .28+51^{\mathrm{d}} 40^{\mathrm{m}} 34^{\mathrm{s}} .9$ & 0.045 & 16.120 & & 1.0 \\
\hline 24 & PG1048+342 & $10^{\mathrm{h}} 51^{\mathrm{m}} 43^{\mathrm{s}} \cdot 90+33^{\mathrm{d}} 59^{\mathrm{m}} 26^{\mathrm{s}} .7$ & 0.167 & 15.810 & & 1.0 \\
\hline 25 & PG1048-090 & $10^{\mathrm{h}} 51^{\mathrm{m}} 29^{\mathrm{s}} \cdot 90-09^{\mathrm{d}} 18^{\mathrm{m}} 10^{\mathrm{s}} .0$ & 0.344 & 16.000 & & 1.0 \\
\hline 26 & PG1049-005 & $10^{\mathrm{h}} 51^{\mathrm{m}} 51^{\mathrm{s}} .44-00^{\mathrm{d}} 51^{\mathrm{m}} 17.7$ & 0.357 & 15.950 & & 1.0 \\
\hline 27 & PG1100+772 & $11^{\mathrm{h}} 04^{\mathrm{m}} 13^{\mathrm{s}} \cdot 69+76^{\mathrm{d}} 58^{\mathrm{m}} 58^{\mathrm{s}} .0$ & 0.313 & 15.860 & & 1.0 \\
\hline 28 & PG1103-006 & $11^{\mathrm{h}} 06^{\mathrm{m}} 31^{\mathrm{s}} .77-00^{\mathrm{d}} 52^{\mathrm{m}} 52^{\mathrm{s}} .5$ & 0.425 & 16.020 & & 1.0 \\
\hline 29 & PG1114+445 & $11^{\mathrm{h}} 17^{\mathrm{m}} 06.40+44^{\mathrm{d}} 13^{\mathrm{m}} 33^{\mathrm{s}} .3$ & 0.144 & 16.050 & & 1.0 \\
\hline 30 & PG1115+407 & $11^{\mathrm{h}} 18^{\mathrm{m}} 30^{\mathrm{s}} .29+40^{\mathrm{d}} 25^{\mathrm{m}} 54^{\mathrm{s}} .0$ & 0.154 & 16.020 & & 1.0 \\
\hline 31 & PG1116+215 & $11^{\mathrm{h}} 19^{\mathrm{m}} 08^{\mathrm{s}} \cdot 68+21^{\mathrm{d}} 19^{\mathrm{m}} 18^{\mathrm{s}} .0$ & 0.177 & 15.170 & & 1.0 \\
\hline 32 & PG1119+120 & $11^{\mathrm{h}} 21^{\mathrm{m}} 47^{\mathrm{s}} \cdot 10+11^{\mathrm{d}} 44^{\mathrm{m}} 18^{\mathrm{s}} \cdot 3$ & 0.049 & 14.650 & & 1.0 \\
\hline 33 & PG1121+422 & $11^{\mathrm{h}} 24^{\mathrm{m}} 39^{\mathrm{s}} \cdot 18+42^{\mathrm{d}} 01^{\mathrm{m}} 45^{\mathrm{s}} \cdot 0$ & 0.234 & 16.020 & & 1.0 \\
\hline 34 & PG1126-041 & $11^{\mathrm{h}} 29^{\mathrm{m}} 16^{\mathrm{s}} .66-04^{\mathrm{d}} 24^{\mathrm{m}} 07^{\mathrm{s}} .6$ & 0.060 & 15.430 & & 1.0 \\
\hline 35 & PG1149-110 & $11^{\mathrm{h}} 52^{\mathrm{m}} 03.54-11^{\mathrm{d}} 22^{\mathrm{m}} 24.3$ & 0.049 & 15.460 & & 1.0 \\
\hline 36 & PG1151+117 & $11^{\mathrm{h}} 53^{\mathrm{m}} 49.27+11^{\mathrm{d}} 28^{\mathrm{m}} 30^{\mathrm{s}} 4$ & 0.176 & 15.510 & & 1.0 \\
\hline 37 & PG1202+281 & $12^{\mathrm{h}} 04^{\mathrm{m}} 42^{\mathrm{s}} .11+27^{\mathrm{d}} 54^{\mathrm{m}} 11^{\mathrm{s}} .8$ & 0.165 & 15.020 & & 1.0 \\
\hline 38 & PG1211+143 & $12^{\mathrm{h}} 14^{\mathrm{m}} 17^{\mathrm{s}} \cdot 70+14^{\mathrm{d}} 03^{\mathrm{m}} 12^{\mathrm{s}} \cdot 6$ & 0.085 & 14.630 & & 1.0 \\
\hline 39 & PG1216+069 & $12^{\mathrm{h}} 19^{\mathrm{m}} 20.93+06^{\mathrm{d}} 38^{\mathrm{m}} 38^{\mathrm{s}} .5$ & 0.334 & 15.680 & & 1.0 \\
\hline 40 & PG1226+023 & $12^{\mathrm{h}} 29^{\mathrm{m}} 06^{\mathrm{s}} .70+02^{\mathrm{d}} 03^{\mathrm{m}} 08^{\mathrm{s}} .6$ & 0.158 & 12.860 & & 1.0 \\
\hline 41 & PG1229+204 & $12^{\mathrm{h}} 32^{\mathrm{m}} 03^{\mathrm{s}} \cdot 60+20^{\mathrm{d}} 09^{\mathrm{m}} 29^{\mathrm{s}} .2$ & 0.064 & 14.650 & & 1.0 \\
\hline 42 & PG1244+026 & $12^{\mathrm{h}} 46^{\mathrm{m}} 35^{\mathrm{s}} \cdot 25+02^{\mathrm{d}} 22^{\mathrm{m}} 08^{\mathrm{s}} .8$ & 0.048 & 16.150 & & 1.0 \\
\hline 43 & PG1259+593 & $13^{\mathrm{h}} 01^{\mathrm{m}} 12^{\mathrm{s}} .93+59^{\mathrm{d}} 02^{\mathrm{m}} 06^{\mathrm{s}} .7$ & 0.472 & 15.600 & & 1.0 \\
\hline 44 & PG1302-102 & $13^{\mathrm{h}} 05^{\mathrm{m}} 33^{\mathrm{s}} .01-10^{\mathrm{d}} 33^{\mathrm{m}} 19^{\mathrm{s}} \cdot 4$ & 0.286 & 15.090 & & 1.0 \\
\hline 45 & PG1307+085 & $13^{\mathrm{h}} 09^{\mathrm{m}} 47^{\mathrm{s}} \cdot 00+08^{\mathrm{d}} 19^{\mathrm{m}} 48^{\mathrm{s}} \cdot 2$ & 0.155 & 15.280 & & 1.0 \\
\hline 46 & PG1309+355 & $13^{\mathrm{h}} 12^{\mathrm{m}} 17^{\mathrm{s}} \cdot 77+35^{\mathrm{d}} 15^{\mathrm{m}} 21^{\mathrm{s}} .2$ & 0.184 & 15.450 & & 1.0 \\
\hline 47 & PG1310-108 & $13^{\mathrm{h}} 13^{\mathrm{m}} 05^{\mathrm{s}} .78-11^{\mathrm{d}} 07^{\mathrm{m}} 42^{\mathrm{s}} .4$ & 0.035 & 15.550 & & 1.0 \\
\hline 48 & PG1322+659 & $13^{\mathrm{h}} 23^{\mathrm{m}} 49.52+65^{\mathrm{d}} 41^{\mathrm{m}} 48^{\mathrm{s}} .2$ & 0.168 & 15.860 & & 1.0 \\
\hline 49 & PG1341+258 & $13^{\mathrm{h}} 43^{\mathrm{m}} 56^{\mathrm{s}} .75+25^{\mathrm{d}} 38^{\mathrm{m}} 47^{\mathrm{s}} .7$ & 0.087 & 15.930 & & 1.0 \\
\hline 50 & PG1351+236 & $13^{\mathrm{h}} 54^{\mathrm{m}} 06^{\mathrm{s}} \cdot 43+23^{\mathrm{d}} 25^{\mathrm{m}} 49^{\mathrm{s}} .1$ & 0.055 & 15.870 & & 1.0 \\
\hline 51 & PG1351+640 & $13^{\mathrm{h}} 53^{\mathrm{m}} 15^{\mathrm{s}} .83+63^{\mathrm{d}} 45^{\mathrm{m}} 45^{\mathrm{s}} .7$ & 0.087 & 15.420 & & 1.0 \\
\hline 52 & PG1352+183 & $13^{\mathrm{h}} 54^{\mathrm{m}} 35^{\mathrm{s}} \cdot 69+18^{\mathrm{d}} 05^{\mathrm{m}} 17^{\mathrm{s}} .5$ & 0.158 & 15.710 & & 1.0 \\
\hline 53 & PG1354+213 & $13^{\mathrm{h}} 56^{\mathrm{m}} 32^{\mathrm{s}} .80+21^{\mathrm{d}} 03^{\mathrm{m}} 52^{\mathrm{s}} \cdot 4$ & 0.300 & 15.850 & & 1.0 \\
\hline 54 & PG1402+261 & $14^{\mathrm{h}} 05^{\mathrm{m}} 16^{\mathrm{s}} .21+25^{\mathrm{d}} 55^{\mathrm{m}} 34^{\mathrm{s}} .1$ & 0.164 & 15.570 & & 1.0 \\
\hline 55 & PG1404+226 & $14^{\mathrm{h}} 06^{\mathrm{m}} 21^{\mathrm{s}} .89+22^{\mathrm{d}} 23^{\mathrm{m}} 46^{\mathrm{s}} \cdot 6$ & 0.098 & 15.820 & & 1.0 \\
\hline 56 & PG1411+442 & $14^{\mathrm{h}} 13^{\mathrm{m}} 48^{\mathrm{s}} \cdot 33+44^{\mathrm{d}} 00^{\mathrm{m}} 14^{\mathrm{s}} .0$ & 0.089 & 14.990 & & 1.0 \\
\hline 57 & PG1415+451 & $14^{\mathrm{h}} 17^{\mathrm{m}} 00^{\mathrm{s}} \cdot 70+44^{\mathrm{d}} 56^{\mathrm{m}} 06^{\mathrm{s}} .0$ & 0.114 & 15.740 & & 1.0 \\
\hline 58 & PG1416-129 & $14^{\mathrm{h}} 19^{\mathrm{m}} 03^{\mathrm{s}} .80-13^{\mathrm{d}} 10^{\mathrm{m}} 44^{\mathrm{s}} .0$ & 0.129 & 15.400 & & 1.0 \\
\hline 59 & PG1425+267 & $14^{\mathrm{h}} 27^{\mathrm{m}} 35^{\mathrm{s}} \cdot 61+26^{\mathrm{d}} 32^{\mathrm{m}} 14^{\mathrm{s}} .5$ & 0.366 & 15.670 & & 1.0 \\
\hline 60 & PG1426+015 & $14^{\mathrm{h}} 29^{\mathrm{m}} 06^{\mathrm{s}} .59+01^{\mathrm{d}} 17^{\mathrm{m}} 06^{\mathrm{s}} .5$ & 0.086 & 15.050 & & 1.0 \\
\hline 61 & PG1427+480 & $14^{\mathrm{h}} 29^{\mathrm{m}} 43^{\mathrm{s}} \cdot 07+47^{\mathrm{d}} 47^{\mathrm{m}} 26^{\mathrm{s}} \cdot 2$ & 0.221 & 16.330 & & 1.0 \\
\hline 62 & PG1435-067 & $14^{\mathrm{h}} 38^{\mathrm{m}} 16^{\mathrm{s}} \cdot 16-06^{\mathrm{d}} 58^{\mathrm{m}} 21^{\mathrm{s}} \cdot 3$ & 0.129 & 15.540 & & 1.0 \\
\hline 63 & PG1440+356 & $14^{\mathrm{h}} 42^{\mathrm{m}} 07^{\mathrm{s}} \cdot 46+35^{\mathrm{d}} 26^{\mathrm{m}} 22^{\mathrm{s}} .9$ & 0.077 & 15.000 & & 1.0 \\
\hline 64 & PG1444+407 & $14^{\mathrm{h}} 46^{\mathrm{m}} 45^{\mathrm{s}} .94+40^{\mathrm{d}} 35^{\mathrm{m}} 05^{\mathrm{s}} .8$ & 0.267 & 15.950 & & 1.0 \\
\hline 65 & PG1448+273 & $14^{\mathrm{h}} 51^{\mathrm{m}} 08^{\mathrm{s}} .76+27^{\mathrm{d}} 09^{\mathrm{m}} 26^{\mathrm{s}} .9$ & 0.065 & 15.010 & & 1.0 \\
\hline
\end{tabular}


Table 1

(Continued)

\begin{tabular}{|c|c|c|c|c|c|c|}
\hline $\begin{array}{l}\text { Number } \\
\text { (1) }\end{array}$ & $\begin{array}{l}\text { Name } \\
(2)\end{array}$ & $\begin{array}{c}\text { Position(J2000) } \\
\text { (3) }\end{array}$ & $\begin{array}{c}z \\
(4)\end{array}$ & $\begin{array}{l}m_{B} \\
(5)\end{array}$ & $\begin{array}{l}m_{K} \\
(6)\end{array}$ & $\begin{array}{l}\text { Type } \\
\text { (7) }\end{array}$ \\
\hline 66 & PG1501+106 & $15^{\mathrm{h}} 04^{\mathrm{m}} 01^{\mathrm{s}} .20+10^{\mathrm{d}} 26^{\mathrm{m}} 16^{\mathrm{s}} .2$ & 0.036 & 15.090 & & 1.0 \\
\hline 67 & PG1512+370 & $15^{\mathrm{h}} 14^{\mathrm{m}} 43^{\mathrm{s}} \cdot 04+36^{\mathrm{d}} 50^{\mathrm{m}} 50^{\mathrm{s}} \cdot 4$ & 0.371 & 15.970 & & 1.0 \\
\hline 68 & PG1519+226 & $15^{\mathrm{h}} 21^{\mathrm{m}} 14^{\mathrm{s}} .26+22^{\mathrm{d}} 27^{\mathrm{m}} 43^{\mathrm{s}} .9$ & 0.137 & 16.090 & & 1.0 \\
\hline 69 & PG1534+580 & $15^{\mathrm{h}} 35^{\mathrm{m}} 52^{\mathrm{s}} \cdot 36+57^{\mathrm{d}} 54^{\mathrm{m}} 09^{\mathrm{s}} .2$ & 0.030 & 15.540 & & 1.0 \\
\hline 70 & PG1535+547 & $15^{\mathrm{h}} 36^{\mathrm{m}} 38^{\mathrm{s}} \cdot 36+54^{\mathrm{d}} 33^{\mathrm{m}} 33^{\mathrm{s}} \cdot 2$ & 0.038 & 15.310 & & 1.0 \\
\hline 71 & PG1543+489 & $15^{\mathrm{h}} 45^{\mathrm{m}} 30^{\mathrm{s}} .24+48^{\mathrm{d}} 46^{\mathrm{m}} 09^{\mathrm{s}} .1$ & 0.400 & 16.050 & & 1.0 \\
\hline 72 & PG1545+210 & $15^{\mathrm{h}} 47^{\mathrm{m}} 43^{\mathrm{s}} \cdot 54+20^{\mathrm{d}} 52^{\mathrm{m}} 16^{\mathrm{s}} \cdot 6$ & 0.266 & 16.050 & & 1.0 \\
\hline 73 & PG1552+085 & $15^{\mathrm{h}} 54^{\mathrm{m}} 44^{\mathrm{s}} .58+08^{\mathrm{d}} 22^{\mathrm{m}} 21^{\mathrm{s}} .5$ & 0.119 & 16.020 & & 1.0 \\
\hline 74 & PG1612+261 & $16^{\mathrm{h}} 14^{\mathrm{m}} 13^{\mathrm{s}} \cdot 20+26^{\mathrm{d}} 04^{\mathrm{m}} 16^{\mathrm{s}} \cdot 2$ & 0.131 & 16.000 & & 1.0 \\
\hline 75 & PG1613+658 & $16^{\mathrm{h}} 13^{\mathrm{m}} 57^{\mathrm{s}} .18+65^{\mathrm{d}} 43^{\mathrm{m}} 09^{\mathrm{s}} .6$ & 0.129 & 15.370 & & 1.0 \\
\hline 76 & PG1617+175 & $16^{\mathrm{h}} 20^{\mathrm{m}} 11^{\mathrm{s}} .29+17^{\mathrm{d}} 24^{\mathrm{m}} 27^{\mathrm{s}} .7$ & 0.114 & 15.530 & & 1.0 \\
\hline 77 & PG1626+554 & $16^{\mathrm{h}} 27^{\mathrm{m}} 56^{\mathrm{s}} .12+55^{\mathrm{d}} 22^{\mathrm{m}} 31^{\mathrm{s}} .5$ & 0.133 & 16.170 & & 1.0 \\
\hline 78 & PG1700+518 & $17^{\mathrm{h}} 01^{\mathrm{m}} 24^{\mathrm{s}} .80+51^{\mathrm{d}} 49^{\mathrm{m}} 20^{\mathrm{s}} .0$ & 0.282 & 15.430 & & 1.0 \\
\hline 79 & PG1704+608 & $17^{\mathrm{h}} 04^{\mathrm{m}} 41^{\mathrm{s}} .38+60^{\mathrm{d}} 44^{\mathrm{m}} 30^{\mathrm{s}} .5$ & 0.371 & 15.900 & & 1.0 \\
\hline 80 & PG2112+059 & $21^{\mathrm{h}} 14^{\mathrm{m}} 52^{\mathrm{s}} .57+06^{\mathrm{d}} 07^{\mathrm{m}} 42^{\mathrm{s}} .5$ & 0.466 & 15.520 & & 1.0 \\
\hline 81 & PG2130+099 & $21^{\mathrm{h}} 32^{\mathrm{m}} 27^{\mathrm{s}} .81+10^{\mathrm{d}} 08^{\mathrm{m}} 19^{\mathrm{s}} .5$ & 0.061 & 14.620 & & 1.0 \\
\hline 82 & PG2209+184 & $22^{\mathrm{h}} 11^{\mathrm{m}} 53^{\mathrm{s}} \cdot 89+18^{\mathrm{d}} 41^{\mathrm{m}} 49^{\mathrm{s}} .9$ & 0.070 & 15.860 & & 1.0 \\
\hline 83 & PG2214+139 & $22^{\mathrm{h}} 17^{\mathrm{m}} 12^{\mathrm{s}} \cdot 26+14^{\mathrm{d}} 14^{\mathrm{m}} 20^{\mathrm{s}} \cdot 9$ & 0.067 & 14.980 & & 1.0 \\
\hline 84 & PG2233+134 & $22^{\mathrm{h}} 36^{\mathrm{m}} 07^{\mathrm{s}} \cdot 68+13^{\mathrm{d}} 43^{\mathrm{m}} 55^{\mathrm{s}} \cdot 3$ & 0.325 & 16.040 & & 1.0 \\
\hline 85 & PG2251+113 & $22^{\mathrm{h}} 54^{\mathrm{m}} 10^{\mathrm{s}} \cdot 40+11^{\mathrm{d}} 36^{\mathrm{m}} 38^{\mathrm{s}} 3$ & 0.323 & 16.250 & & 1.0 \\
\hline 86 & PG2304+042 & $23^{\mathrm{h}} 07^{\mathrm{m}} 02^{\mathrm{s}} \cdot 91+04^{\mathrm{d}} 32^{\mathrm{m}} 57^{\mathrm{s}} .2$ & 0.042 & 15.440 & & 1.0 \\
\hline 87 & PG2308+098 & $23^{\mathrm{h}} 11^{\mathrm{m}} 17^{\mathrm{s}} .76+10^{\mathrm{d}} 08^{\mathrm{m}} 15^{\mathrm{s}} .5$ & 0.432 & 16.120 & & 1.0 \\
\hline 88 & 2MASSiJ000703.6+155423 & $00^{\mathrm{h}} 07^{\mathrm{m}} 03^{\mathrm{s}} \cdot 60+15 \mathrm{~d} 54^{\mathrm{m}} 23^{\mathrm{s}} .8$ & 0.114 & & 13.100 & 1.8 \\
\hline 89 & 2MASSiJ000810.8+135452 & $00^{\mathrm{h}} 08^{\mathrm{m}} 10^{\mathrm{s}} .86+13^{\mathrm{d}} 54^{\mathrm{m}} 52^{\mathrm{s}} \cdot 3$ & 0.185 & & 14.400 & 2.0 \\
\hline 90 & 2MASSiJ004118.7+281640 & $00^{\mathrm{h}} 41^{\mathrm{m}} 18^{\mathrm{s}} \cdot 70+28^{\mathrm{d}} 16^{\mathrm{m}} 40^{\mathrm{s}} .0$ & 0.194 & & 12.500 & 1.0 \\
\hline 91 & 2MASSiJ005010.1+280619 & $00^{\mathrm{h}} 50^{\mathrm{m}} 10^{\mathrm{s}} .05+28^{\mathrm{d}} 06^{\mathrm{m}} 20^{\mathrm{s}} .7$ & 0.277 & & 15.130 & \\
\hline 92 & 2MASSiJ005055.7+293328 & $00^{\mathrm{h}} 50^{\mathrm{m}} 55^{\mathrm{s}} \cdot 70+29^{\mathrm{d}} 33^{\mathrm{m}} 28^{\mathrm{s}} \cdot 1$ & 0.136 & & 13.220 & 2.0 \\
\hline 93 & 2MASSiJ010230.1+262337 & $01^{\mathrm{h}} 02^{\mathrm{m}} 30^{\mathrm{s}} \cdot 10+26^{\mathrm{d}} 23^{\mathrm{m}} 37^{\mathrm{s}} .0$ & 0.194 & & 13.830 & 1.0 \\
\hline 94 & 2MASSiJ010835.1+214818 & $01^{\mathrm{h}} 08^{\mathrm{m}} 35^{\mathrm{s}} \cdot 10+21^{\mathrm{d}} 48^{\mathrm{m}} 18^{\mathrm{s}} .0$ & 0.285 & & 13.460 & 1.9 \\
\hline 95 & 2MASSiJ012031.5+200327 & $01^{\mathrm{h}} 20^{\mathrm{m}} 31^{\mathrm{s}} .50+20^{\mathrm{d}} 03^{\mathrm{m}} 27^{\mathrm{s}} .0$ & 0.087 & & 12.470 & \\
\hline 96 & 2MASSiJ015721.0+171248 & $01^{\mathrm{h}} 57^{\mathrm{m}} 21^{\mathrm{s}} .00+17^{\mathrm{d}} 12^{\mathrm{m}} 48^{\mathrm{s}} .0$ & 0.213 & & 13.160 & 1.0 \\
\hline 97 & 2MASSiJ022150.6+132741 & $02^{\mathrm{h}} 21^{\mathrm{m}} 50^{\mathrm{s}} \cdot 60+13^{\mathrm{d}} 27^{\mathrm{m}} 40^{\mathrm{s}} 8$ & 0.140 & & 13.250 & 1.8 \\
\hline 98 & 2MASSiJ024807.3+145957 & $02^{\mathrm{h}} 48^{\mathrm{m}} 07^{\mathrm{s}} \cdot 36+14^{\mathrm{d}} 59^{\mathrm{m}} 57^{\mathrm{s}} \cdot 7$ & 0.072 & & 12.650 & 1.0 \\
\hline 99 & 2MASSiJ034857.6+125547 & $03^{\mathrm{h}} 48^{\mathrm{m}} 57^{\mathrm{s}} \cdot 68+12^{\mathrm{d}} 55^{\mathrm{m}} 47^{\mathrm{s}} .4$ & 0.210 & & 13.600 & 1.0 \\
\hline 100 & 2MASSiJ081652.2+425829 & $08^{\mathrm{h}} 16^{\mathrm{m}} 52^{\mathrm{s}} .24+42^{\mathrm{d}} 58^{\mathrm{m}} 29^{\mathrm{s}} .4$ & 0.235 & & 13.730 & 1.0 \\
\hline 101 & 2MASSiJ082311.3+435318 & $08^{\mathrm{h}} 23^{\mathrm{m}} 11^{\mathrm{s}} .27+43^{\mathrm{d}} 53^{\mathrm{m}} 18^{\mathrm{s}} .5$ & 0.182 & & 12.850 & 1.5 \\
\hline 102 & 2MASSiJ091848.6+211717 & $09^{\mathrm{h}} 18^{\mathrm{m}} 48^{\mathrm{s}} \cdot 61+21^{\mathrm{d}} 17^{\mathrm{m}} 17^{\mathrm{s}} \cdot 0$ & 0.149 & & 12.550 & 1.5 \\
\hline 103 & 2MASSiJ092049.0+190320 & $09^{\mathrm{h}} 20^{\mathrm{m}} 49^{\mathrm{s}} \cdot 00+19^{\mathrm{d}} 03^{\mathrm{m}} 20^{\mathrm{s}} \cdot 0$ & 0.156 & & 14.920 & 1.0 \\
\hline 104 & 2MASSiJ094636.4+205610 & $09^{\mathrm{h}} 46^{\mathrm{m}} 36^{\mathrm{s}} \cdot 43+20^{\mathrm{d}} 56^{\mathrm{m}} 11^{\mathrm{s}} .0$ & 0.280 & & 13.720 & 1.5 \\
\hline 105 & 2MASSiJ095504.5+170556 & $09^{\mathrm{h}} 55^{\mathrm{m}} 04^{\mathrm{s}} .55+17^{\mathrm{d}} 05^{\mathrm{m}} 56.4$ & 0.139 & & 13.440 & 1.0 \\
\hline 106 & 2MASSiJ100121.1+215011 & $10^{\mathrm{h}} 01^{\mathrm{m}} 21^{\mathrm{s}} \cdot 17+21^{\mathrm{d}} 50^{\mathrm{m}} 11^{\mathrm{s}} \cdot 2$ & 0.248 & & 14.680 & \\
\hline 107 & 2MASSiJ101400.4+194614 & $10^{\mathrm{h}} 13^{\mathrm{m}} 58^{\mathrm{s}} .99+19^{\mathrm{d}} 45^{\mathrm{m}} 59^{\mathrm{s}} .1$ & 0.110 & & 12.370 & 1.5 \\
\hline 108 & 2MASSiJ101649.3+215435 & $10^{\mathrm{h}} 16^{\mathrm{m}} 49^{\mathrm{s}} \cdot 35+21^{\mathrm{d}} 54^{\mathrm{m}} 35^{\mathrm{s}} .0$ & 0.257 & & 13.940 & 1.0 \\
\hline 109 & 2MASSiJ102724.9+121920 & $10^{\mathrm{h}} 27^{\mathrm{m}} 24^{\mathrm{s}} .98+12^{\mathrm{d}} 19^{\mathrm{m}} 19.8$ & 0.231 & & 13.220 & 1.5 \\
\hline 110 & 2MASSiJ105144.2+353930 & $10^{\mathrm{h}} 51^{\mathrm{m}} 44^{\mathrm{s}} .24+35^{\mathrm{d}} 39^{\mathrm{m}} 30^{\mathrm{s}} .8$ & 0.158 & & 13.540 & \\
\hline 111 & 2MASSiJ125807.4+232921 & $12^{\mathrm{h}} 58^{\mathrm{m}} 07^{\mathrm{s}} \cdot 46+23^{\mathrm{d}} 29^{\mathrm{m}} 21^{\mathrm{s}} \cdot 6$ & 0.259 & & 13.450 & 1.0 \\
\hline 112 & 2MASSiJ130005.3+163214 & $13^{\mathrm{h}} 00^{\mathrm{m}} 05^{\mathrm{s}} \cdot 35+16^{\mathrm{d}} 32^{\mathrm{m}} 14^{\mathrm{s}} .8$ & 0.080 & & 11.860 & 1.0 \\
\hline 113 & 2MASSiJ130700.6+233805 & $13^{\mathrm{h}} 07^{\mathrm{m}} 00^{\mathrm{s}} .63+23^{\mathrm{d}} 38^{\mathrm{m}} 05^{\mathrm{s}} .2$ & 0.275 & & 13.450 & 1.0 \\
\hline 114 & 2MASSiJ132917.5+121340 & $13^{\mathrm{h}} 29^{\mathrm{m}} 17^{\mathrm{s}} .52+12^{\mathrm{d}} 13^{\mathrm{m}} 40^{\mathrm{s}} \cdot 2$ & 0.203 & & 14.120 & 1.0 \\
\hline 115 & 2MASSiJ134915.2+220032 & $13^{\mathrm{h}} 49^{\mathrm{m}} 15^{\mathrm{s}} \cdot 20+22^{\mathrm{d}} 00^{\mathrm{m}} 32^{\mathrm{s}} \cdot 7$ & 0.062 & & 12.240 & 1.5 \\
\hline 116 & 2MASSiJ1402511+263117 & $14^{\mathrm{h}} 02^{\mathrm{m}} 51^{\mathrm{s}} .20+26^{\mathrm{d}} 31^{\mathrm{m}} 17^{\mathrm{s}} .6$ & 0.187 & & 12.670 & 1.0 \\
\hline 117 & 2MASSiJ145331.5+135358 & $14^{\mathrm{h}} 53^{\mathrm{m}} 31^{\mathrm{s}} .52+13^{\mathrm{d}} 53^{\mathrm{m}} 58^{\mathrm{s}} .7$ & 0.139 & & 13.090 & 1.0 \\
\hline 118 & 2MASSiJ145406.6+195028 & $14^{\mathrm{h}} 54^{\mathrm{m}} 06^{\mathrm{s}} .68+19^{\mathrm{d}} 50^{\mathrm{m}} 28^{\mathrm{s}} .2$ & 0.260 & & 14.710 & 1.5 \\
\hline 119 & 2MASSiJ145410.1+195648 & $14^{\mathrm{h}} 54^{\mathrm{m}} 10^{\mathrm{s}} .17+19^{\mathrm{d}} 56^{\mathrm{m}} 48^{\mathrm{s}} .7$ & 0.243 & & 14.170 & 1.9 \\
\hline 120 & 2MASSiJ145608.6+275008 & $14^{\mathrm{h}} 56^{\mathrm{m}} 08^{\mathrm{s}} .65+27^{\mathrm{d}} 50^{\mathrm{m}} 08^{\mathrm{s}} .8$ & 0.250 & & 13.380 & 1.0 \\
\hline 121 & 2MASSiJ150113.1+232908 & $15^{\mathrm{h}} 01^{\mathrm{m}} 13^{\mathrm{s}} \cdot 20+23^{\mathrm{d}} 29^{\mathrm{m}} 08^{\mathrm{s}} \cdot 3$ & 0.258 & & 13.460 & 1.0 \\
\hline 122 & 2MASSiJ151621.1+225944 & $15^{\mathrm{h}} 16^{\mathrm{m}} 21^{\mathrm{s}} .11+22^{\mathrm{d}} 59^{\mathrm{m}} 44^{\mathrm{s}} .7$ & 0.190 & & 14.120 & 1.0 \\
\hline 123 & 2MASSiJ151653.2+190048 & $15^{\mathrm{h}} 16^{\mathrm{m}} 53^{\mathrm{s}} .23+19^{\mathrm{d}} 00^{\mathrm{m}} 48^{\mathrm{s}} .3$ & 0.190 & & 11.410 & 1.0 \\
\hline 124 & 2MASSiJ151901.5+183804 & $15^{\mathrm{h}} 19^{\mathrm{m}} 01^{\mathrm{s}} .48+18^{\mathrm{d}} 38^{\mathrm{m}} 04^{\mathrm{s}} .9$ & 0.187 & & 14.250 & 1.0 \\
\hline 125 & 2MASSiJ152151.0+225120 & $15^{\mathrm{h}} 21^{\mathrm{m}} 51^{\mathrm{s}} .00+22^{\mathrm{d}} 51^{\mathrm{m}} 20^{\mathrm{s}} .0$ & 0.287 & & 14.300 & 1.0 \\
\hline 126 & 2MASSiJ154307.7+193751 & $15^{\mathrm{h}} 43^{\mathrm{m}} 07^{\mathrm{s}} .78+19^{\mathrm{d}} 37^{\mathrm{m}} 51^{\mathrm{s}} .8$ & 0.228 & & 12.740 & 1.5 \\
\hline 127 & 2MASSiJ163700.2+222114 & $16^{\mathrm{h}} 37^{\mathrm{m}} 00^{\mathrm{s}} .22+22^{\mathrm{d}} 21^{\mathrm{m}} 14^{\mathrm{s}} .1$ & 0.211 & & 13.590 & 1.0 \\
\hline 128 & 2MASSiJ163736.5+254302 & $16^{\mathrm{h}} 37^{\mathrm{m}} 36^{\mathrm{s}} .52+25^{\mathrm{d}} 43^{\mathrm{m}} 02^{\mathrm{s}} .8$ & 0.277 & & 14.170 & 1.9 \\
\hline 129 & 2MASSiJ165939.7+183436 & $16^{\mathrm{h}} 59^{\mathrm{m}} 39^{\mathrm{s}} \cdot 77+18^{\mathrm{d}} 34^{\mathrm{m}} 36^{\mathrm{s}} .8$ & 0.170 & & 12.910 & 1.5 \\
\hline 130 & 2MASSiJ170536.6+210137 & $17^{\mathrm{h}} 05^{\mathrm{m}} 36^{\mathrm{s}} \cdot 66+21^{\mathrm{d}} 01^{\mathrm{m}} 38^{\mathrm{s}} .0$ & 0.271 & & 14.310 & 1.0 \\
\hline
\end{tabular}


Table 1

(Continued)

\begin{tabular}{|c|c|c|c|c|c|c|}
\hline $\begin{array}{l}\text { Number } \\
\text { (1) }\end{array}$ & $\begin{array}{l}\text { Name } \\
(2)\end{array}$ & $\begin{array}{l}\text { Position(J2000) } \\
\text { (3) }\end{array}$ & $\begin{array}{c}z \\
(4)\end{array}$ & $\begin{array}{l}m_{B} \\
(5)\end{array}$ & $\begin{array}{l}m_{K} \\
(6)\end{array}$ & $\begin{array}{l}\text { Type } \\
\text { (7) }\end{array}$ \\
\hline 131 & 2MASSiJ171442.7+260248 & $17^{\mathrm{h}} 14^{\mathrm{m}} 42^{\mathrm{s}} \cdot 77+26^{\mathrm{d}} 02^{\mathrm{m}} 48^{\mathrm{s}} \cdot 6$ & 0.163 & & 13.080 & 1.0 \\
\hline 132 & 2MASSiJ222221.1+195947 & $22^{\mathrm{h}} 22^{\mathrm{m}} 21^{\mathrm{s}} \cdot 14+19^{\mathrm{d}} 59^{\mathrm{m}} 47^{\mathrm{s}} .1$ & 0.211 & & 12.920 & 1.0 \\
\hline 133 & 2MASSiJ222554.2+195837 & $22^{\mathrm{h}} 25^{\mathrm{m}} 54^{\mathrm{s}} .25+19^{\mathrm{d}} 58^{\mathrm{m}} 37^{\mathrm{s}} .2$ & 0.147 & & 13.490 & 2.0 \\
\hline 134 & 2MASSiJ223742.6+145614 & $22^{\mathrm{h}} 37^{\mathrm{m}} 42^{\mathrm{s}} \cdot 60+14^{\mathrm{d}} 56^{\mathrm{m}} 14^{\mathrm{s}} .0$ & 0.277 & & 14.000 & 1.0 \\
\hline 135 & 2MASSiJ223946.0+192955 & $22^{\mathrm{h}} 39^{\mathrm{m}} 46^{\mathrm{s}} .00+19^{\mathrm{d}} 29^{\mathrm{m}} 55^{\mathrm{s}} .0$ & 0.194 & & 14.670 & \\
\hline 136 & 2MASSiJ230304.3+162440 & $23^{\mathrm{h}} 03^{\mathrm{m}} 04^{\mathrm{s}} \cdot 30+16^{\mathrm{d}} 24^{\mathrm{m}} 40^{\mathrm{s}} \cdot 0$ & 0.289 & & 14.670 & 2.0 \\
\hline 137 & 2MASSiJ230442.4+270616 & $23^{\mathrm{h}} 04^{\mathrm{m}} 42^{\mathrm{s}} \cdot 40+27^{\mathrm{d}} 06^{\mathrm{m}} 16^{\mathrm{s}} .0$ & 0.237 & & 14.770 & 1.5 \\
\hline 138 & 2MASSiJ234259.3+134750 & $23^{\mathrm{h}} 42^{\mathrm{m}} 59^{\mathrm{s}} \cdot 36+13^{\mathrm{d}} 47^{\mathrm{m}} 50^{\mathrm{s}} \cdot 4$ & 0.299 & & 14.190 & 1.5 \\
\hline 139 & 2MASSiJ234449.5+122143 & $23^{\mathrm{h}} 44^{\mathrm{m}} 49^{\mathrm{s}} \cdot 56+12^{\mathrm{d}} 21^{\mathrm{m}} 43^{\mathrm{s}} .1$ & 0.199 & & 12.910 & 1.0 \\
\hline
\end{tabular}

Notes. Column 2: the name of the target; Column 3: the position of the target; Column 4: redshift; Column 5: $B$-band magnitude of PG quasars in Vega system (see Schmidt \& Green 1983); Column 6: $K_{s}$ magnitude of 2MASS quasars in internal 2MASS magnitude system (http://www.ipac.caltech.edu/2mass/releases/allsky/doc/sec6_4a.html). Column 7: the optical type of emission line where 1.0 for broad emission line, 2.0 for narrow emission line and values between 1.0 and 2.0 for intermediate types.

quasars. These data were reduced to images as described in Balog et al. (2013). First, bad and saturated pixels were flagged. The response was calibrated and corrected for flat fields. A highpass filter was applied to eliminate the $1 / f$ noise of the detector. Aperture photometry was carried out on the reduced images as in Balog et al. (2013). For objects with both Spitzer $160 \mu \mathrm{m}$ and Herschel $160 \mu \mathrm{m}$ detections, the latter is used because of (generally) higher signal to noise and greater freedom from structured sky emission.

\section{SPECTRAL DECOMPOSITIONS}

\subsection{Fitting Spectral Features}

The IR spectra of quasars are rich in features. As shown in Figure 1, they usually contain strong hot dust continuum emission, cold dust emission, silicate emission or absorption features at 9.7 and $18 \mu \mathrm{m}$, aromatic features mainly at 6.2, 7.7, $8.6,11.3$, and $12.1 \mu \mathrm{m}$, plus atomic fine-structure and molecular hydrogen emission lines.

Although these features can be measured individually, we prefer to fit them simultaneously so that blends can be separated. Our method is based on a simple physical model, similar to the IDL program PAHFIT.pro which was developed by Smith et al. (2007) to decompose the spectra of normal galaxies. The dust continuum is modeled through a combination of a series of blackbodies at fixed temperatures of 1000, 580, 415, 210, 97, $58,48,41,32,29$, and $26 \mathrm{~K}$ while letting the normalization be free. The highest temperature is chosen to fit the hottest dust emission peaking around $3 \mu \mathrm{m}$, and the lowest temperature is limited by our SED coverage that in general does not extend beyond $200 \mu \mathrm{m}$. This set of temperatures was based on several test runs, and includes criteria such as the peak wavelengths of the blackbodies should not lie within spectral regions of strong and broad silicate and aromatic features. The emission at wavelengths longer than $200 \mu \mathrm{m}$ is usually described by a modified blackbody. However, as we have little $>200 \mu \mathrm{m}$ photometry and are not fitting features in this spectral range, we simply adopted blackbody spectra. Higher-fidelity fits to these long wavelengths are discussed in the following section. Each silicate feature either in emission or absorption is modeled through two Gaussian functions: (1) for the $9.7 \mu \mathrm{m}$ silicate feature, central wavelengths of two Gaussians were set to be 10.0 and $11.5 \mu \mathrm{m}$ that can vary by $\pm 3 \%$. The standard deviations (i.e., widths) were set to be $8 \%$ and $10 \%$ of the central wavelengths, respectively. The fractional Gaussian widths were allowed to vary from $-2 \%$ to $+4 \%$. The normalizations of the two Gaussians are free, with the initial value set to be half of the difference between the observed flux at the Gaussian central wavelength and the one at $8 \mu \mathrm{m}$; and (2) for the $18 \mu \mathrm{m}$ feature, two Gaussians were placed at 16.0 and $19.0 \mu \mathrm{m}$, respectively, with all other parameters treated similarly to those for the $9.7 \mu \mathrm{m}$ feature; the initial value of the normalization is set to be half of the difference between the observed flux at the Gaussian central wavelength and the one at $15 \mu \mathrm{m}$. Figure 2 shows examples of the two-Gaussian fitting for the 9.7 silicate feature. A single Gaussian function was used to model the emission lines including $\mathrm{H}_{2} S(3) 9.67 \mu \mathrm{m}$, [SIV] $10.52 \mu \mathrm{m}$, [Ne II] $12.81 \mu \mathrm{m}$, [Ne III] $15.56 \mu \mathrm{m}, \mathrm{H}_{2} S(1) 17.03 \mu \mathrm{m}$, and [O IV] $25.91 \mu \mathrm{m}$. The line width of the Gaussian was set by the spectral resolution at a given wavelength but allowed to vary by $\pm 20 \%$, while the normalization was totally free. As detailed in Smith et al. (2007), the aromatic features were described by Drude profiles with fixed centers and widths but free normalizations.

The fitting results from minimizing $\chi^{2}$ were in general excellent under visual inspection. However, for about $20 \%$ of the sample, the broad silicate emission features appeared to mimic dust blackbody emission. In these cases, the initial value of the silicate feature strength needs to be relatively accurate and was obtained by spline interpolation or visual estimate. We used a general criterion that the dust continuum underlying the silicate emission feature between 9 and $20 \mu \mathrm{m}$ should change gradually and monotonically.

The results of the SED decompositions are shown in Figure 1. With the fitted profiles, various features are quantified and listed in Table 4. The silicate feature strength is defined as $\ln \left(f^{\text {peak }} / f_{\text {cont }}^{\text {peak }}\right)$, where $f^{\text {peak }}$ is the flux density at the wavelength where the silicate emission feature peaks or the silicate absorption feature shows a minimum based on the two fitted Gaussian profiles for the individual features, and $f_{\text {cont }}^{\text {peak }}$ is the continuum flux density at the same wavelength. The continuum luminosities at given wavelengths were measured as the average of the spectra over a $2 \mu \mathrm{m}$ range around the central wavelengths. All the listed errors in Table 4 only consider the photon noise in the spectrum. For the silicate feature intensity, due to the difficulty in differentiating the broad feature from the underlying continuum, there are additional systematic uncertainties that could be up to $0.1-0.2$. The peak wavelength can also suffer systematic 
Table 2

Broad-band Photometry of the Sample

\begin{tabular}{|c|c|c|c|c|c|c|c|}
\hline Number & Name & $\begin{array}{c}f_{\text {Spitzer }-24 \mu \mathrm{m}} \\
(\mathrm{mJy}) \\
(3)\end{array}$ & $\begin{array}{c}f_{\text {Spitzer }-70 \mu \mathrm{m}} \\
(\mathrm{mJy}) \\
(4)\end{array}$ & $\begin{array}{c}f_{\text {Spitzer-160 } \mu \mathrm{m}} \\
(\mathrm{mJy}) \\
(5)\end{array}$ & $\begin{array}{c}f_{\text {Herschel-160 }} \mu \mathrm{m} \\
(\mathrm{mJy}) \\
(6)\end{array}$ & $\begin{array}{c}\text { ClRS-SL }_{\text {IRS-LL }} \\
\text { (7) }\end{array}$ & $\begin{array}{c}\mathrm{C}_{\mathrm{IRS}}^{\mathrm{MIPS}} \\
(8)\end{array}$ \\
\hline 1 & PG0003+158 & $24.69 \pm 0.38$ & $21.26 \pm 4.29$ & $<115.88$ & $<40.68$ & 1.31 & 0.99 \\
\hline 2 & PG0003+199 & $-9.00 \pm 1.00$ & $329.80 \pm 11.0$ & & $138.06 \pm 12.3$ & & 1.77 \\
\hline 3 & PG0007+106 & $139.90 \pm 0.29$ & $210.80 \pm 9.31$ & $194.40 \pm 49.1$ & $260.19 \pm 14.8$ & 1.00 & 0.92 \\
\hline 4 & PG0026+129 & $46.82 \pm 0.37$ & $<33.67$ & $<112.96$ & & 1.29 & 1.13 \\
\hline 5 & PG0043+039 & $27.08 \pm 0.41$ & $38.53 \pm 7.63$ & $<60.59$ & $89.71 \pm 13.76$ & 0.95 & 1.18 \\
\hline 6 & PG0049+171 & $17.46 \pm 0.49$ & $<14.48$ & $<163.63$ & $<40.56$ & 1.00 & 1.06 \\
\hline 7 & PG0050+124 & $953.40 \pm 0.48$ & $2084.00 \pm 11$ & $1612.00 \pm 34$ & $1884.34 \pm 17$ & 0.96 & 1.06 \\
\hline 8 & PG0052+251 & $71.19 \pm 0.33$ & $82.07 \pm 8.68$ & $<150.31$ & $155.53 \pm 16.2$ & 0.98 & 0.99 \\
\hline 9 & PG0157+001 & $-9.00 \pm 1.00$ & & & $1301.47 \pm 11$ & 1.01 & 1.35 \\
\hline 10 & PG0804+761 & $199.10 \pm 0.31$ & $122.70 \pm 5.71$ & $<68.58$ & $50.07 \pm 13.64$ & 0.95 & 0.99 \\
\hline 11 & PG0838+770 & $75.25 \pm 0.27$ & $140.60 \pm 6.82$ & $117.70 \pm 22.3$ & $224.59 \pm 12.5$ & 0.94 & 1.15 \\
\hline 12 & PG0844+349 & $98.72 \pm 0.37$ & $100.90 \pm 6.75$ & $163.80 \pm 28.3$ & $239.90 \pm 14.5$ & 0.93 & 1.15 \\
\hline 13 & PG0921+525 & $93.44 \pm 0.31$ & $95.75 \pm 10.03$ & & $142.30 \pm 10.2$ & 0.91 & 1.16 \\
\hline 14 & PG0923+201 & $54.05 \pm 0.41$ & $50.00 \pm 4.82$ & $<227.46$ & $63.26 \pm 12.65$ & 0.89 & 1.13 \\
\hline 15 & PG0923+129 & $242.50 \pm 0.43$ & & & $1059.21 \pm 14$ & 0.94 & 1.11 \\
\hline 16 & PG0934+013 & $86.50 \pm 0.39$ & $214.60 \pm 10.8$ & & $305.10 \pm 15.0$ & 0.94 & 1.12 \\
\hline 17 & PG0947+396 & $50.11 \pm 0.36$ & $107.80 \pm 9.89$ & & $189.65 \pm 10.8$ & 0.89 & 1.09 \\
\hline 18 & PG0953+414 & $46.15 \pm 0.34$ & $39.49 \pm 10.73$ & $<95.10$ & $69.89 \pm 12.99$ & 0.98 & 1.08 \\
\hline 19 & PG1001+054 & $38.50 \pm 0.40$ & $39.85 \pm 5.94$ & $<89.45$ & $56.30 \pm 10.60$ & 0.87 & 1.13 \\
\hline 20 & PG1004+130 & $80.60 \pm 0.50$ & $104.20 \pm 3.99$ & $<119.74$ & $82.72 \pm 15.23$ & 1.00 & 1.10 \\
\hline 21 & PG1011-040 & $104.00 \pm 0.33$ & $179.80 \pm 6.96$ & $266.60 \pm 40.4$ & $233.90 \pm 13.7$ & 1.00 & 1.08 \\
\hline 22 & PG1012+008 & $57.18 \pm 0.72$ & $93.63 \pm 7.90$ & $175.60 \pm 31.1$ & $104.81 \pm 17.1$ & 1.00 & 1.07 \\
\hline 23 & PG1022+519 & $46.62 \pm 0.29$ & $246.90 \pm 7.94$ & & $288.98 \pm 11.5$ & 0.94 & 1.08 \\
\hline 24 & PG1048+342 & $26.97 \pm 0.36$ & $62.60 \pm 10.17$ & $133.10 \pm 18.2$ & $160.10 \pm 13.1$ & 1.06 & 1.14 \\
\hline 25 & PG1048-090 & $25.31 \pm 0.35$ & $57.32 \pm 6.75$ & & $<56.84$ & 1.20 & 1.23 \\
\hline 26 & PG1049-005 & $101.90 \pm 0.37$ & $259.80 \pm 6.27$ & $<77.58$ & $170.00 \pm 12.5$ & 1.00 & 1.03 \\
\hline 27 & PG1100+772 & $48.81 \pm 0.26$ & $61.16 \pm 2.66$ & $<154.62$ & $90.04 \pm 16.89$ & 1.00 & 1.07 \\
\hline 28 & PG1103-006 & $37.95 \pm 0.35$ & $55.29 \pm 6.97$ & $<143.80$ & $49.97 \pm 10.70$ & 1.00 & 1.16 \\
\hline 29 & PG1114+445 & $137.20 \pm 0.33$ & $88.37 \pm 6.91$ & $<60.59$ & $<42.98$ & 1.00 & 1.08 \\
\hline 30 & PG1115+407 & $44.98 \pm 0.32$ & $189.60 \pm 7.34$ & $267.30 \pm 23.9$ & $303.31 \pm 11.7$ & 1.00 & 1.11 \\
\hline 31 & PG1116+215 & $103.70 \pm 0.45$ & $64.96 \pm 10.16$ & $<81.10$ & & 1.05 & 0.93 \\
\hline 32 & PG1119+120 & $235.70 \pm 0.35$ & $351.70 \pm 7.97$ & $334.20 \pm 36.7$ & $346.25 \pm 11.8$ & 1.00 & 1.08 \\
\hline 33 & PG1121+422 & $14.50 \pm 0.34$ & $<10.49$ & $<71.47$ & $<39.66$ & 0.97 & 1.19 \\
\hline 34 & PG1126-041 & $261.90 \pm 0.49$ & $495.20 \pm 10.5$ & $349.90 \pm 49.7$ & $451.76 \pm 13.3$ & 1.75 & 1.05 \\
\hline 35 & PG1149-110 & $119.40 \pm 0.41$ & $220.50 \pm 14.3$ & & $349.89 \pm 12.7$ & 0.94 & 1.04 \\
\hline 36 & PG1151+117 & $32.28 \pm 0.41$ & $36.19 \pm 5.75$ & $<108.66$ & $<33.61$ & 1.06 & 1.16 \\
\hline 37 & PG1202+281 & $82.08 \pm 0.36$ & $85.88 \pm 13.59$ & & $91.88 \pm 13.24$ & 1.00 & 1.05 \\
\hline 38 & PG1211+143 & $250.90 \pm 0.39$ & $139.80 \pm 8.98$ & $<114.47$ & $<41.63$ & 1.02 & 0.99 \\
\hline 39 & PG1216+069 & $28.21 \pm 0.41$ & $<22.52$ & & $<42.43$ & 0.82 & 1.22 \\
\hline 40 & PG1226+023 & $592.50 \pm 0.39$ & $985.90 \pm 8.64$ & $1010.00 \pm 26$ & & 0.98 & 0.95 \\
\hline 41 & PG1229+204 & $155.50 \pm 0.34$ & $152.40 \pm 6.64$ & $198.60 \pm 31.1$ & $250.75 \pm 12.1$ & 1.00 & 1.15 \\
\hline 42 & PG1244+026 & $108.90 \pm 0.40$ & $192.80 \pm 9.34$ & $235.80 \pm 35.9$ & $109.60 \pm 12.4$ & 0.95 & 1.11 \\
\hline 43 & PG1259+593 & $22.29 \pm 0.28$ & $22.94 \pm 3.86$ & $<48.54$ & $<41.71$ & 0.97 & 1.07 \\
\hline 44 & PG1302-102 & $99.66 \pm 0.37$ & $121.10 \pm 8.30$ & $<136.95$ & $184.77 \pm 13.8$ & 0.95 & 1.09 \\
\hline 45 & PG1307+085 & $70.81 \pm 0.35$ & $86.30 \pm 9.18$ & $<107.19$ & $63.89 \pm 11.51$ & 1.00 & 0.97 \\
\hline 46 & PG1309+355 & $108.90 \pm 0.30$ & $45.33 \pm 9.19$ & $<56.05$ & $132.10 \pm 13.9$ & 1.22 & 0.98 \\
\hline 47 & PG1310-108 & $126.60 \pm 0.40$ & $105.10 \pm 7.37$ & $<128.36$ & & 0.93 & 1.12 \\
\hline 48 & PG1322+659 & $48.75 \pm 0.39$ & $149.60 \pm 8.80$ & $<87.11$ & $136.28 \pm 14.9$ & 0.97 & 1.13 \\
\hline 49 & PG1341+258 & $47.07 \pm 0.33$ & $102.80 \pm 8.71$ & & $75.77 \pm 13.27$ & 0.99 & 1.18 \\
\hline 50 & PG1351+236 & $53.75 \pm 0.37$ & $369.80 \pm 7.64$ & $375.60 \pm 21.2$ & $491.88 \pm 11.7$ & 1.01 & 0.98 \\
\hline 51 & PG1351+640 & $437.90 \pm 0.45$ & $626.10 \pm 13.4$ & $455.30 \pm 46.4$ & $336.70 \pm 16.9$ & 0.96 & 1.04 \\
\hline 52 & PG1352+183 & $32.28 \pm 0.34$ & $18.18 \pm 4.84$ & $<71.84$ & $<39.22$ & 5.37 & 1.05 \\
\hline 53 & PG1354+213 & $30.65 \pm 0.31$ & $51.89 \pm 5.18$ & $134.80 \pm 37.6$ & $<40.59$ & 1.04 & 1.12 \\
\hline 54 & PG1402+261 & $106.40 \pm 0.33$ & $223.80 \pm 8.69$ & $186.10 \pm 30.9$ & $173.77 \pm 13.9$ & 1.00 & 1.00 \\
\hline 55 & PG1404+226 & $27.96 \pm 0.33$ & $47.12 \pm 5.98$ & $<73.61$ & $110.07 \pm 13.9$ & 1.02 & 1.03 \\
\hline 56 & PG1411+442 & $123.80 \pm 0.28$ & $140.80 \pm 6.12$ & $94.89 \pm 16.63$ & $179.71 \pm 15.3$ & 1.14 & 1.07 \\
\hline 57 & PG1415+451 & $62.08 \pm 0.31$ & $106.10 \pm 6.18$ & $105.60 \pm 17.6$ & $138.19 \pm 14.2$ & 1.00 & 1.06 \\
\hline 58 & PG1416-129 & $28.26 \pm 0.38$ & $29.88 \pm 6.43$ & $<143.85$ & $38.74 \pm 11.96$ & 1.10 & 1.24 \\
\hline 59 & PG1425+267 & $49.08 \pm 0.30$ & $82.70 \pm 2.71$ & $<72.31$ & $71.25 \pm 14.28$ & 0.95 & 1.15 \\
\hline 60 & PG1426+015 & $189.90 \pm 0.37$ & $301.90 \pm 9.89$ & $298.60 \pm 32.2$ & $344.06 \pm 13.9$ & 0.95 & 1.12 \\
\hline 61 & PG1427+480 & $44.80 \pm 0.28$ & $109.10 \pm 9.12$ & $<66.01$ & $51.73 \pm 10.71$ & 1.09 & 1.11 \\
\hline 62 & PG1435-067 & $40.27 \pm 0.37$ & $49.23 \pm 4.01$ & $<84.19$ & $<36.63$ & 0.90 & 1.08 \\
\hline 63 & PG1440+356 & $184.90 \pm 0.31$ & $807.10 \pm 10.1$ & $597.50 \pm 31.1$ & $628.43 \pm 16.6$ & 0.95 & 1.11 \\
\hline 64 & PG1444+407 & $62.21 \pm 0.28$ & $90.14 \pm 9.13$ & $<95.16$ & & 0.99 & 1.04 \\
\hline
\end{tabular}


Table 2

(Continued)

\begin{tabular}{|c|c|c|c|c|c|c|c|}
\hline Number & Name & $\begin{array}{c}f_{\text {Spitzer-24 }} \mu \mathrm{m} \\
(\mathrm{mJy}) \\
\text { (3) }\end{array}$ & $\begin{array}{c}f_{\text {Spitzer-70 } \mu \mathrm{m}} \\
(\mathrm{mJy}) \\
(4)\end{array}$ & $\begin{array}{c}f_{\text {Spitzer-160 } \mu \mathrm{m}} \\
(\mathrm{mJy}) \\
(5)\end{array}$ & $\begin{array}{c}f_{\text {Herschel }-160 \mu \mathrm{m}} \\
(\mathrm{mJy}) \\
(6)\end{array}$ & $\begin{array}{c}\text { ClRS-LL }_{\text {IRS-SL }} \\
(7)\end{array}$ & $\mathrm{C}_{\mathrm{IRS}}^{\mathrm{MIPS}}$ \\
\hline 65 & PG1448+273 & $108.20 \pm 0.31$ & $133.20 \pm 7.76$ & $156.60 \pm 25.8$ & $181.71 \pm 12.4$ & 0.92 & 1.20 \\
\hline 66 & PG1501+106 & $433.50 \pm 0.41$ & $365.00 \pm 8.96$ & $135.40 \pm 18.6$ & & 1.01 & 1.02 \\
\hline 67 & PG1512+370 & $33.86 \pm 0.28$ & $43.34 \pm 3.86$ & $<68.37$ & $64.35 \pm 11.06$ & 0.98 & 1.17 \\
\hline 68 & PG1519+226 & $61.93 \pm 0.32$ & $84.57 \pm 6.48$ & $<107.36$ & $88.82 \pm 11.01$ & 0.96 & 1.05 \\
\hline 69 & PG1534+580 & $178.80 \pm 0.28$ & $178.00 \pm 4.76$ & $125.10 \pm 20.2$ & & 0.97 & 1.07 \\
\hline 70 & PG1535+547 & $77.58 \pm 0.29$ & $96.20 \pm 5.91$ & & $114.81 \pm 15.4$ & 1.07 & 1.16 \\
\hline 71 & PG1543+489 & $105.50 \pm 0.45$ & $303.80 \pm 6.96$ & $261.40 \pm 32.8$ & $206.31 \pm 12.2$ & 0.86 & 1.10 \\
\hline 72 & PG1545+210 & $35.84 \pm 0.29$ & $37.85 \pm 2.13$ & $<102.97$ & $<38.30$ & 1.04 & 1.01 \\
\hline 73 & PG1552+085 & $29.89 \pm 0.35$ & $31.16 \pm 4.51$ & $<58.00$ & $<43.01$ & 1.00 & 1.08 \\
\hline 74 & PG1612+261 & $99.88 \pm 0.34$ & $211.90 \pm 6.71$ & $121.60 \pm 39.7$ & $229.89 \pm 14.4$ & 0.93 & 1.09 \\
\hline 75 & PG1613+658 & $-9.00 \pm 1.00$ & & & $793.21 \pm 16.1$ & 0.95 & 1.51 \\
\hline 76 & PG1617+175 & $52.85 \pm 0.34$ & $49.48 \pm 5.32$ & $<93.83$ & $<51.05$ & 1.00 & 1.09 \\
\hline 77 & PG1626+554 & $18.69 \pm 0.30$ & $21.22 \pm 5.40$ & $<73.03$ & & 0.94 & 1.12 \\
\hline 78 & PG1700+518 & $188.10 \pm 0.26$ & $401.30 \pm 4.72$ & $207.30 \pm 27.0$ & $328.60 \pm 12.3$ & 1.11 & 1.07 \\
\hline 79 & PG1704+608 & $109.60 \pm 0.23$ & $205.40 \pm 3.28$ & $135.80 \pm 21.5$ & $109.36 \pm 14.5$ & 1.00 & 1.03 \\
\hline 80 & PG2112+059 & $76.31 \pm 0.31$ & $68.78 \pm 6.19$ & $<84.58$ & $103.80 \pm 9.91$ & 1.00 & 1.14 \\
\hline 81 & PG2130+099 & $312.60 \pm 0.32$ & $486.30 \pm 6.98$ & $330.10 \pm 25.1$ & $397.25 \pm 12.0$ & 1.05 & 1.00 \\
\hline 82 & PG2209+184 & $25.06 \pm 0.68$ & $102.10 \pm 10.5$ & $243.70 \pm 42.1$ & $210.40 \pm 13.7$ & 1.10 & 1.23 \\
\hline 83 & PG2214+139 & $98.17 \pm 0.33$ & $103.50 \pm 8.46$ & & $136.31 \pm 10.1$ & 0.98 & 1.13 \\
\hline 84 & PG2233+134 & $59.86 \pm 0.35$ & $93.84 \pm 10.12$ & $<177.06$ & $85.11 \pm 10.14$ & 0.94 & 1.10 \\
\hline 85 & PG2251+113 & $46.80 \pm 0.35$ & $104.70 \pm 7.98$ & $<114.14$ & $77.57 \pm 13.83$ & 0.95 & 1.08 \\
\hline 86 & PG2304+042 & $26.77 \pm 0.42$ & $<29.95$ & & & 1.07 & 1.02 \\
\hline 87 & PG2308+098 & $25.75 \pm 0.35$ & $<30.94$ & & $<48.33$ & 0.88 & 1.12 \\
\hline 88 & 2MASSiJ000703.6+155423 & $68.95 \pm 0.37$ & $262.80 \pm 7.86$ & $258.50 \pm 51.4$ & & 0.93 & 1.04 \\
\hline 89 & 2MASSiJ000810.8+135452 & $8.81 \pm 0.96$ & $39.34 \pm 7.25$ & $<186.76$ & & & \\
\hline 90 & 2MASSiJ004118.7+281640 & $78.74 \pm 0.37$ & $108.10 \pm 10.1$ & $149.40 \pm 43.5$ & & 1.14 & 1.05 \\
\hline 91 & 2MASSiJ005010.1+280619 & $10.28 \pm 0.32$ & $134.80 \pm 6.52$ & $207.80 \pm 53.7$ & & 1.11 & 1.46 \\
\hline 92 & 2MASSiJ005055.7+293328 & $61.67 \pm 0.31$ & $199.70 \pm 6.09$ & $<83.01$ & & 1.11 & 1.04 \\
\hline 93 & 2MASSiJ010230.1+262337 & $18.27 \pm 0.32$ & $33.31 \pm 7.11$ & $<80.71$ & & 1.19 & 1.09 \\
\hline 94 & 2MASSiJ010835.1+214818 & $78.77 \pm 0.35$ & $107.30 \pm 10.7$ & $<128.46$ & & 1.09 & 1.04 \\
\hline 95 & 2MASSiJ012031.5+200327 & $7.50 \pm 0.39$ & $92.06 \pm 8.82$ & & & 0.87 & 2.25 \\
\hline 96 & 2MASSiJ015721.0+171248 & $33.50 \pm 0.36$ & $240.30 \pm 14.3$ & $<343.97$ & & 0.97 & 1.03 \\
\hline 97 & 2MASSiJ022150.6+132741 & $115.30 \pm 0.39$ & $411.50 \pm 7.96$ & $<179.55$ & & 1.82 & 1.03 \\
\hline 98 & 2MASSiJ024807.3+145957 & $84.15 \pm 0.43$ & $177.60 \pm 9.95$ & $<101.16$ & & 1.03 & 1.03 \\
\hline 99 & 2MASSiJ034857.6+125547 & $157.30 \pm 0.39$ & $302.70 \pm 13.1$ & $275.20 \pm 80.1$ & & 1.29 & 1.04 \\
\hline 100 & 2MASSiJ081652.2+425829 & $12.19 \pm 0.34$ & $<11.89$ & $<106.08$ & & 0.96 & 1.17 \\
\hline 101 & 2MASSiJ082311.3+435318 & $78.20 \pm 0.34$ & $222.50 \pm 6.12$ & $283.80 \pm 28.0$ & & 1.04 & 1.04 \\
\hline 102 & 2MASSiJ091848.6+211717 & $96.67 \pm 0.37$ & $205.00 \pm 10.9$ & $181.50 \pm 32.8$ & & 1.00 & 0.96 \\
\hline 103 & 2MASSiJ092049.0+190320 & $13.45 \pm 0.35$ & $<23.70$ & $<76.02$ & & 1.04 & 1.34 \\
\hline 104 & 2MASSiJ094636.4+205610 & $45.70 \pm 0.40$ & $40.78 \pm 9.56$ & $<121.49$ & & 1.06 & 1.05 \\
\hline 105 & 2MASSiJ095504.5+170556 & $20.95 \pm 0.41$ & $16.25 \pm 4.97$ & $<92.72$ & & 1.10 & 1.10 \\
\hline 106 & 2MASSiJ100121.1+215011 & $19.52 \pm 0.40$ & $225.40 \pm 9.41$ & $226.10 \pm 37.4$ & & 2.00 & 1.16 \\
\hline 107 & 2MASSiJ101400.4+194614 & $86.69 \pm 0.38$ & $143.20 \pm 8.57$ & & & & \\
\hline 108 & 2MASSiJ101649.3+215435 & $23.37 \pm 0.39$ & $42.89 \pm 7.75$ & $<74.29$ & & 1.27 & 0.92 \\
\hline 109 & 2MASSiJ102724.9+121920 & $86.35 \pm 0.41$ & $99.53 \pm 9.68$ & & & 1.38 & 1.05 \\
\hline 110 & 2MASSiJ105144.2+353930 & $35.66 \pm 0.35$ & $53.64 \pm 7.95$ & & & 1.00 & 1.05 \\
\hline 111 & 2MASSiJ125807.4+232921 & $72.69 \pm 0.33$ & $129.60 \pm 7.32$ & $<84.02$ & & 1.02 & 0.96 \\
\hline 112 & 2MASSiJ130005.3+163214 & $162.50 \pm 0.37$ & $68.99 \pm 7.36$ & $<74.69$ & & 1.00 & 0.99 \\
\hline 113 & 2MASSiJ130700.6+233805 & $78.32 \pm 0.32$ & $796.30 \pm 7.37$ & $395.60 \pm 36.8$ & & 1.56 & 1.03 \\
\hline 114 & 2MASSiJ132917.5+121340 & $13.37 \pm 0.88$ & $<30.72$ & & & 1.26 & 1.23 \\
\hline 115 & 2MASSiJ134915.2+220032 & $200.10 \pm 0.35$ & $413.40 \pm 6.19$ & $227.40 \pm 31.7$ & & & \\
\hline 116 & 2MASSiJ1402511+263117 & $26.99 \pm 0.29$ & $29.89 \pm 4.37$ & $<62.76$ & & 1.04 & 1.11 \\
\hline 117 & 2MASSiJ145331.5+135358 & $124.80 \pm 0.31$ & $702.10 \pm 7.51$ & $424.00 \pm 26.3$ & & 0.94 & 0.96 \\
\hline 118 & 2MASSiJ145406.6+195028 & $15.99 \pm 0.29$ & $36.17 \pm 5.57$ & $<79.24$ & & 1.32 & 1.13 \\
\hline 119 & 2MASSiJ145410.1+195648 & $32.34 \pm 0.32$ & $38.69 \pm 6.66$ & $<121.89$ & & 1.29 & 1.26 \\
\hline 120 & 2MASSiJ145608.6+275008 & $31.06 \pm 0.29$ & $96.27 \pm 7.40$ & $141.70 \pm 31.9$ & & 1.31 & 1.18 \\
\hline 121 & 2MASSiJ150113.1+232908 & $51.13 \pm 0.30$ & $112.60 \pm 17.1$ & $213.40 \pm 53.4$ & & 0.96 & 1.07 \\
\hline 122 & 2MASSiJ151621.1+225944 & $20.61 \pm 0.33$ & $31.61 \pm 8.18$ & $<130.20$ & & 1.25 & 1.06 \\
\hline 123 & 2MASSiJ151653.2+190048 & $135.80 \pm 0.31$ & $82.47 \pm 9.66$ & $95.02 \pm 27.06$ & & 1.02 & 0.98 \\
\hline 124 & 2MASSiJ151901.5+183804 & $10.44 \pm 0.31$ & $22.44 \pm 2.57$ & $<79.79$ & & 0.94 & 1.23 \\
\hline 125 & 2MASSiJ152151.0+225120 & $55.28 \pm 0.33$ & $574.70 \pm 8.31$ & $637.90 \pm 36.8$ & & 1.10 & 1.02 \\
\hline 126 & 2MASSiJ154307.7+193751 & $87.04 \pm 0.28$ & $120.90 \pm 9.78$ & $<79.39$ & & 1.03 & 1.02 \\
\hline 127 & 2MASSiJ163700.2+222114 & $25.44 \pm 0.67$ & $146.30 \pm 14.7$ & $255.80 \pm 30.9$ & & 1.17 & 0.42 \\
\hline 128 & 2MASSiJ163736.5+254302 & $40.33 \pm 0.25$ & $86.63 \pm 7.84$ & $<97.47$ & & 0.95 & 1.10 \\
\hline
\end{tabular}


Table 2

(Continued)

\begin{tabular}{|c|c|c|c|c|c|c|c|}
\hline Number & $\begin{array}{l}\text { Name } \\
(2)\end{array}$ & $\begin{array}{c}f_{\text {Spitzer-24 }} \mu \mathrm{m} \\
(\mathrm{mJy}) \\
(3)\end{array}$ & $\begin{array}{c}f_{\text {Spitzer-70 } \mu \mathrm{m}} \\
(\mathrm{mJy}) \\
(4)\end{array}$ & $\begin{array}{c}f_{\text {Spitzer-160 }} \mu \mathrm{m} \\
(\mathrm{mJy}) \\
(5)\end{array}$ & $\begin{array}{c}f_{\text {Herschel-160 }} \mu \mathrm{m} \\
(\mathrm{mJy}) \\
(6)\end{array}$ & $\begin{array}{c}\text { CIRS-SL }_{\text {IRS-LL }} \\
(7)\end{array}$ & $\begin{array}{r}\mathrm{C}_{\mathrm{IRS}}^{\mathrm{MIPS}} \\
(8)\end{array}$ \\
\hline 129 & 2MASSiJ165939.7+183436 & $140.70 \pm 0.28$ & $269.40 \pm 8.26$ & & & 1.00 & 0.96 \\
\hline 130 & 2MASSiJ170536.6+210137 & $11.84 \pm 0.32$ & $74.79 \pm 3.19$ & $<111.69$ & & 0.90 & 1.05 \\
\hline 131 & 2MASSiJ171442.7+260248 & $26.09 \pm 0.32$ & $75.63 \pm 3.35$ & $<76.58$ & & 1.13 & 1.40 \\
\hline 132 & 2MASSiJ222221.1+195947 & $51.96 \pm 0.30$ & $67.19 \pm 10.07$ & & & 1.08 & 1.10 \\
\hline 133 & 2MASSiJ222554.2+195837 & $45.09 \pm 0.33$ & $166.70 \pm 5.17$ & $<113.14$ & & 0.92 & 1.00 \\
\hline 134 & 2MASSiJ223742.6+145614 & $20.75 \pm 0.33$ & $91.35 \pm 7.19$ & & & 1.14 & 1.14 \\
\hline 135 & 2MASSiJ223946.0+192955 & $7.61 \pm 0.30$ & $70.38 \pm 6.57$ & & & 0.87 & 2.12 \\
\hline 136 & 2MASSiJ230304.3+162440 & $4.66 \pm 0.33$ & $104.50 \pm 8.25$ & & & 0.88 & 1.00 \\
\hline 137 & 2MASSiJ230442.4+270616 & $5.63 \pm 0.33$ & $33.15 \pm 6.39$ & $<90.77$ & & 0.97 & 1.28 \\
\hline 138 & 2MASSiJ234259.3+134750 & $19.63 \pm 0.41$ & $47.88 \pm 6.63$ & $<93.73$ & & 1.13 & 1.15 \\
\hline 139 & 2MASSiJ234449.5+122143 & $59.40 \pm 0.35$ & $152.60 \pm 8.76$ & $<109.66$ & & 1.04 & 1.05 \\
\hline
\end{tabular}

Note. The factor in Column 7 is used to multiply with the IRS short-low to long-low, while the factor in Column 8 is used to scale the IRS to the MIPS $24 \mu \mathrm{m}$ photometry.

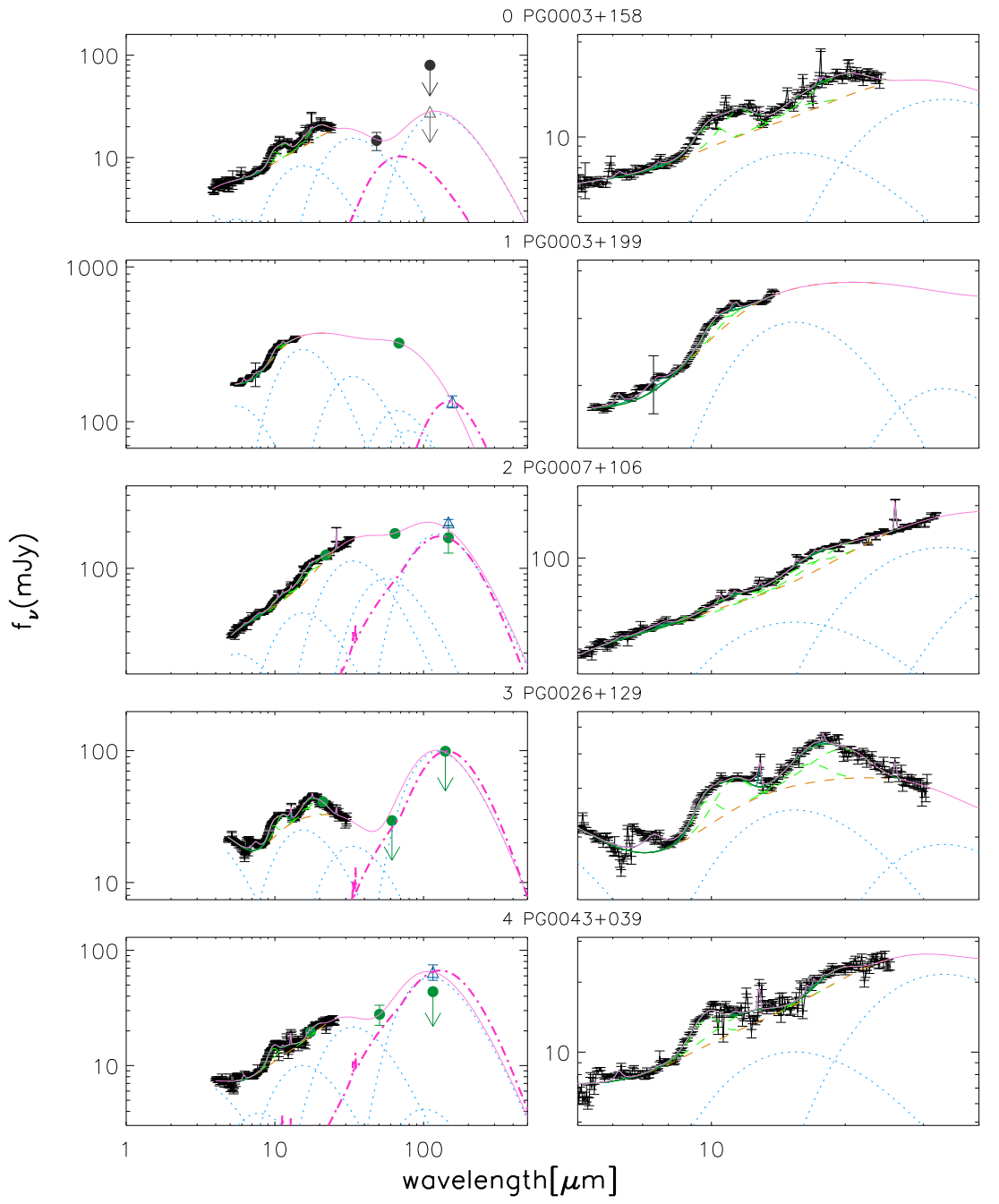

Figure 1. Rest-frame IRS spectra (black points) and broad-band photometry (green filled circles). For each object, the left panel shows the full SEDs while the right panel zooms into the 5-40 $\mu \mathrm{m}$ spectral range. The pink solid lines are the fitted curves as a sum of all individual fitted profiles including blackbody, silicate features, aromatic features, and emission lines. The blue dotted lines indicate the fitted individual blackbody emission the sum of which is shown as yellow dashed line. The green dashed lines represent the fitted silicate feature profiles. The pink dotted-dashed line is the best-fit star-forming template derived from the library of Rieke et al. (2009).

(A color version and an extended version of this figure are available in the online journal.) 

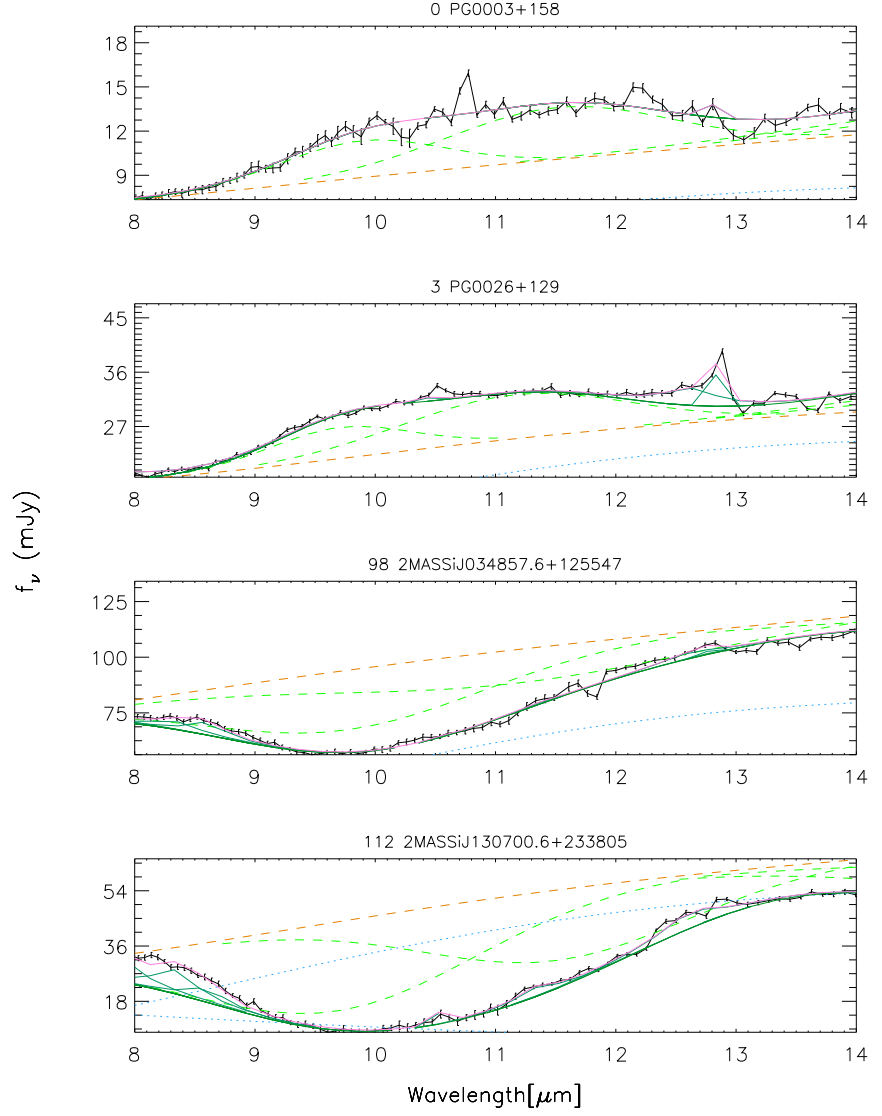

Figure 2. Examples of two Gaussian fitting for the $9.7 \mu \mathrm{m}$ silicate feature. All line styles are the same as the Figure 1.

(A color version of this figure is available in the online journal.)

errors of $\sim 1 \mu \mathrm{m}$ for the $9.7 \mu \mathrm{m}$ feature and $2 \mu \mathrm{m}$ for the $18 \mu \mathrm{m}$ silicate feature.

\subsection{Decomposing SEDs into Star-forming and Dusty Torus Components}

The above spectral decomposition aims to measure intensities of different spectral features. In this section we decompose the full IR SED into emission from SF and the radiation from the dusty torus. For an initial reconnaissance, we assume the emission at a far-IR wavelength or an aromatic feature comes entirely from star-forming regions and then select the starforming template (Rieke et al. 2009) that yields the closest observed fluxes. We have derived such estimates in three ways: (1) $11.3 \mu \mathrm{m}$ aromatic features; (2) MIPS $70 \mu \mathrm{m}$ photometry; and (3) MIPS or PACS $160 \mu \mathrm{m}$ photometry.

Although the aromatic features in quasar spectra arise from SF regions in the host galaxies (e.g., Shi et al. 2007), observations of the central regions $(\sim 0.5 \mathrm{kpc})$ of Seyfert galaxies indicate that the nuclear radiation may suppress the aromatic features at 6.2, 7.7, and $8.6 \mu \mathrm{m}$, but not the $11.3 \mu \mathrm{m}$ feature (Diamond-Stanic \& Rieke 2010; Esquej et al. 2014). We convert the aromatic flux for this feature to SFR using the star-forming templates of Rieke et al. (2009). An issue with this method is the large intrinsic scatter between aromatic fluxes and SFRs as seen in non-AGN star-forming galaxies (Smith et al. 2007; Calzetti et al. 2007).

Far-IR luminosity is generally considered to be a reliable SFR indicator for galaxies. For each broad-band photometry measurement, we choose the star-forming template with monochromatic luminosity closest to the observation, normalize it to the
Table 3

Infrared Spectra and Photometry of the Sample

\begin{tabular}{lcccc}
\hline \hline Number & Name & Wave & $\begin{array}{c}f_{v} \\
(\mathrm{mJy})\end{array}$ & $\begin{array}{c}\text { Error } \\
(\mathrm{mJy})\end{array}$ \\
$(1)$ & $(2)$ & $(3)$ & $(4)$ & $(5)$ \\
\hline 1 & PG0003+158 & 5.52 & 7.01 & 0.43 \\
& & 5.56 & 7.05 & 0.44 \\
& 5.59 & 6.95 & 0.31 \\
& 5.63 & 6.74 & 0.44 \\
& 5.67 & 6.98 & 0.42 \\
\hline
\end{tabular}

(This table is available in its entirety in a machine-readable form in the online journal. A portion is shown here for guidance regarding its form and content.)

measured flux density, and estimate the far-IR luminosity from this normalized template. The risk for this approach is that the far-IR emission may be contaminated by cold dust in the dusty torus. After choosing the best star-forming template, including bands not affected by cold interstellar medium (ISM) dust, we measured the SFRs based on the $24 \mu \mathrm{m}$ emission of the template following Rieke et al. (2009).

We evaluate the contribution from the dusty torus to the 70 and $160 \mu \mathrm{m}$ emission by comparing SFRs from these two bands to the aromatic-based SFRs, since the aromatic emission should arise mainly from star-forming regions. Although aromatic features could be excited by B-type stars (Peeters et al. 2004; Vega et al. 2010), for our sample with relatively high far-IR luminosity (mostly above $10^{10} L_{\odot}$ ), such a contribution should be negligible. As shown in Figure 3, no systematic offset is seen between $160 \mu \mathrm{m}$ based SFRs and the SFRs from aromatic features. The situation for the $70 \mu \mathrm{m}$ based SFRs is more complex. For SFRs larger than $10 M_{\odot} \mathrm{yr}^{-1}$ from the aromatic feature, there is no systematic offset. For lower SFRs, the estimates from the $70 \mu \mathrm{m}$ measurement tend to be high. This behavior may indicate contamination of the $70 \mu \mathrm{m}$ photometry (typically at rest-frame $\sim 50 \mu \mathrm{m}$ for our sample) by emission from the dusty torus in cases where the SFR is low.

The above three methods demonstrate the overall consistency of SFRs estimated from monochromatic photometry or a single aromatic feature. Therefore, it is permissible to improve the accuracy in the SFR estimates by combining the three methods through template fitting. For objects with $160 \mu \mathrm{m}$ detections, a first-guess star-forming template is chosen to have the closest $160 \mu \mathrm{m}$ luminosity to the observation. If this template, however, produces higher $70 \mu \mathrm{m}$ luminosity than is observed, we discard it and instead choose the next colder template in the library of Rieke et al. (2009). Sometimes this process needs to be repeated until the best template is chosen. Similarly, for objects with $70 \mu \mathrm{m}$ detections, the best star-forming template is the one that has the closest $70 \mu \mathrm{m}$ luminosity to the observation. However, if the template produces $160 \mu \mathrm{m}$ output above the observed upper limit, a hotter template is chosen. As indicated in Figure 3, for $70 \mu \mathrm{m}$ based SFRs smaller than $10 M_{\odot} \mathrm{yr}^{-1}$, we reduce the SFR estimate by 0.3 dex to account statistically for the torus emission. For objects with aromatic features, the best-fit template is required to not exceed the 70 and $160 \mu \mathrm{m}$ upper limits; if it does it is renormalized to lower its luminosity and make it consistent. The estimated SFRs are listed in Table 4. Both quasar samples have SFR measurements for as many as 94\% of the members, with upper limits for the rest. We assigned a systematic error of 0.3 dex for $160 \mu \mathrm{m}$ based SFRs as well as $70 \mu \mathrm{m}$ based SFRs above $10 M_{\odot} \mathrm{yr}^{-1}$, and $0.5 \mathrm{dex}$ for $70 \mu \mathrm{m}$ based SFRs below $10 M_{\odot} \mathrm{yr}^{-1}$ as well as aromatic based SFRs, 
Table 4

Measured Features and Physical Parameters

\begin{tabular}{|c|c|c|c|c|c|c|c|c|c|c|c|}
\hline ID & Name & $\operatorname{Str}_{9.7 \mu \mathrm{m}-\mathrm{Sil}}$ & $\begin{array}{l}\lambda_{9.7 \mu \mathrm{m}-\mathrm{Sil}}^{\text {peak }} \\
\quad(\mu \mathrm{m})\end{array}$ & $\operatorname{Str}_{18 \mu \mathrm{m}-\mathrm{Sil}}$ & $\begin{array}{c}\lambda_{18 \mu \mathrm{m}-\mathrm{Sil}}^{\text {peak }} \\
(\mu \mathrm{m})\end{array}$ & $\begin{array}{c}\mathrm{EW}_{11.3 \mu \mathrm{mPAH}} \\
(\mu \mathrm{m})\end{array}$ & $\begin{array}{l}\log L_{5 \mu \mathrm{m}} \\
\left(\log \left(L_{\odot}\right)\right)\end{array}$ & $\begin{array}{l}\log L_{15 \mu \mathrm{m}} \\
\left(\log \left(L_{\odot}\right)\right)\end{array}$ & $\begin{array}{l}\log L_{18 \mu \mathrm{m}} \\
\left(\log \left(L_{\odot}\right)\right)\end{array}$ & $\begin{array}{l}\log L_{25 \mu \mathrm{m}} \\
\left(\log \left(L_{\odot}\right)\right)\end{array}$ & $\begin{array}{c}\log (\mathrm{SFR}) \\
\left(M_{\odot} \mathrm{yr}^{-1}\right)\end{array}$ \\
\hline 1 & PG0003+158 & $0.32 \pm 0.01$ & 11.3 & $0.28 \pm 0.03$ & 19.3 & $<0.032$ & 11.81 & 11.74 & 11.78 & 11.63 & $1.43 \pm 0.30$ \\
\hline 2 & PG0003+199 & $0.10 \pm 0.00$ & 10.3 & & & $0.034 \pm 0.002$ & 10.55 & 10.40 & 10.33 & 10.19 & $-0.94 \pm 0.30$ \\
\hline 3 & PG0007+106 & $0.10 \pm 0.02$ & 11.4 & $0.23 \pm 0.01$ & 17.4 & $0.027 \pm 0.007$ & 10.90 & 10.93 & 10.96 & 10.94 & $0.66 \pm 0.30$ \\
\hline 4 & PG0026+129 & $0.30 \pm 0.02$ & 10.1 & $0.30 \pm 0.02$ & 17.8 & $<0.019$ & 11.24 & 10.99 & 10.99 & 10.74 & $<0.65$ \\
\hline 5 & PG0043+039 & $0.33 \pm 0.03$ & 9.8 & $0.13 \pm 0.03$ & 19.1 & $<0.043$ & 11.75 & 11.62 & 11.66 & 11.58 & $1.64 \pm 0.30$ \\
\hline 6 & PG0049+171 & $0.30 \pm 0.03$ & 10.5 & $0.53 \pm 0.03$ & 17.5 & $<0.041$ & 10.05 & 9.92 & 9.92 & 9.61 & $<-0.65$ \\
\hline 7 & PG0050+124 & $0.28 \pm 0.00$ & 10.0 & $0.11 \pm 0.00$ & 17.4 & $0.055 \pm 0.002$ & 11.24 & 11.40 & 11.44 & 11.43 & $1.59 \pm 0.30$ \\
\hline 8 & PG0052+251 & $0.22 \pm 0.04$ & 10.5 & $0.39 \pm 0.02$ & 17.4 & $0.046 \pm 0.014$ & 11.19 & 11.22 & 11.24 & 10.99 & $1.05 \pm 0.30$ \\
\hline 9 & PG0157+001 & $0.25 \pm 0.01$ & 9.9 & $0.22 \pm 0.01$ & 15.7 & $0.106 \pm 0.005$ & 11.52 & 11.98 & 12.06 & 12.28 & $2.50 \pm 0.30$ \\
\hline 10 & PG0804+761 & $0.32 \pm 0.01$ & 10.0 & $0.14 \pm 0.00$ & 20.2 & $0.019 \pm 0.003$ & 11.43 & 11.23 & 11.25 & 11.13 & $-0.09 \pm 0.30$ \\
\hline 11 & PG0838+770 & $0.09 \pm 0.02$ & 10.2 & $0.15 \pm 0.01$ & 17.3 & $0.035 \pm 0.007$ & 10.86 & 11.03 & 11.06 & 11.02 & $1.01 \pm 0.30$ \\
\hline 12 & PG0844+349 & $0.34 \pm 0.02$ & 10.4 & $0.26 \pm 0.02$ & 19.5 & $0.036 \pm 0.008$ & 10.57 & 10.49 & 10.57 & 10.42 & $0.03 \pm 0.30$ \\
\hline 13 & PG0921+525 & $0.25 \pm 0.01$ & 10.9 & $0.58 \pm 0.01$ & 17.7 & $<0.010$ & 10.13 & 10.10 & 10.13 & 9.83 & $-0.63 \pm 0.30$ \\
\hline 14 & PG0923+201 & $0.31 \pm 0.02$ & 10.0 & $0.17 \pm 0.01$ & 19.2 & $<0.028$ & 11.49 & 11.26 & 11.26 & 11.14 & $1.06 \pm 0.30$ \\
\hline 15 & PG0923+129 & $0.17 \pm 0.00$ & 11.8 & $0.35 \pm 0.01$ & 18.3 & $0.085 \pm 0.003$ & 9.98 & 10.21 & 10.25 & 10.14 & $0.22 \pm 0.30$ \\
\hline 16 & PG0934+013 & $0.08 \pm 0.01$ & 11.1 & $0.15 \pm 0.01$ & 18.2 & $0.077 \pm 0.005$ & 10.03 & 10.18 & 10.23 & 10.21 & $0.16 \pm 0.30$ \\
\hline 17 & PG0947+396 & $0.11 \pm 0.02$ & 11.8 & $0.10 \pm 0.01$ & 16.9 & $<0.017$ & 11.38 & 11.30 & 11.30 & 11.23 & $1.39 \pm 0.30$ \\
\hline 18 & PG0953+414 & $0.28 \pm 0.04$ & 10.4 & $0.22 \pm 0.03$ & 19.5 & $<0.052$ & 11.65 & 11.42 & 11.42 & 11.25 & $1.29 \pm 0.30$ \\
\hline 19 & PG1001+054 & $0.24 \pm 0.02$ & 11.5 & $0.22 \pm 0.04$ & 16.6 & $<0.033$ & 11.16 & 10.95 & 10.96 & 10.87 & $0.55 \pm 0.30$ \\
\hline 20 & PG1004+130 & $0.63 \pm 0.01$ & 10.1 & $0.26 \pm 0.01$ & 17.8 & $0.090 \pm 0.006$ & 11.51 & 11.62 & 11.66 & 11.57 & $1.46 \pm 0.30$ \\
\hline 21 & PG1011-040 & $0.38 \pm 0.01$ & 10.7 & $0.34 \pm 0.01$ & 18.1 & $0.062 \pm 0.005$ & 10.27 & 10.44 & 10.49 & 10.37 & $0.23 \pm 0.30$ \\
\hline 22 & PG1012+008 & $0.12 \pm 0.01$ & 11.7 & $0.07 \pm 0.02$ & 18.7 & $<0.021$ & 11.25 & 11.26 & 11.26 & 11.21 & $1.26 \pm 0.30$ \\
\hline 23 & PG1022+519 & $0.11 \pm 0.01$ & 11.2 & $0.17 \pm 0.01$ & 19.2 & $0.236 \pm 0.006$ & 9.86 & 9.84 & 9.88 & 9.83 & $-0.03 \pm 0.30$ \\
\hline 24 & PG1048+342 & $0.09 \pm 0.02$ & 11.8 & $0.21 \pm 0.02$ & 18.4 & $<0.031$ & 10.89 & 10.88 & 10.88 & 10.71 & $1.05 \pm 0.30$ \\
\hline 25 & PG1048-090 & $0.45 \pm 0.02$ & 10.7 & $0.27 \pm 0.03$ & 19.5 & $<0.047$ & 11.62 & 11.48 & 11.49 & 11.41 & $1.55 \pm 0.30$ \\
\hline 26 & PG1049-005 & $0.23 \pm 0.01$ & 10.7 & $0.15 \pm 0.01$ & 16.0 & $<0.013$ & 12.04 & 12.11 & 12.14 & 12.15 & $2.17 \pm 0.30$ \\
\hline 27 & PG1100+772 & $0.31 \pm 0.01$ & 11.1 & $0.17 \pm 0.01$ & 18.9 & $<0.021$ & 11.75 & 11.70 & 11.70 & 11.62 & $1.64 \pm 0.30$ \\
\hline 28 & PG1103-006 & $0.40 \pm 0.01$ & 10.5 & $0.18 \pm 0.02$ & 19.2 & $<0.030$ & 11.88 & 11.89 & 11.89 & 11.81 & $1.76 \pm 0.30$ \\
\hline 29 & PG1114+445 & $0.11 \pm 0.01$ & 11.4 & $0.21 \pm 0.00$ & 18.5 & $<0.008$ & 11.36 & 11.41 & 11.43 & 11.28 & $0.60 \pm 0.50$ \\
\hline 30 & PG1115+407 & $0.14 \pm 0.01$ & 11.9 & $0.16 \pm 0.02$ & 15.9 & $0.138 \pm 0.007$ & 11.06 & 10.99 & 10.98 & 10.95 & $1.51 \pm 0.30$ \\
\hline 31 & PG1116+215 & $0.27 \pm 0.06$ & 10.8 & $0.20 \pm 0.02$ & 19.5 & $<0.051$ & 11.76 & 11.48 & 11.48 & 11.35 & $0.65 \pm 0.50$ \\
\hline 32 & PG1119+120 & $0.15 \pm 0.01$ & 11.8 & $0.18 \pm 0.01$ & 16.6 & $0.035 \pm 0.005$ & 10.35 & 10.63 & 10.65 & 10.62 & $0.18 \pm 0.30$ \\
\hline 33 & PG1121+422 & $0.22 \pm 0.02$ & 11.0 & $0.17 \pm 0.04$ & 19.2 & $<0.043$ & 11.28 & 10.93 & 10.90 & 10.69 & $<0.65$ \\
\hline 34 & PG1126-041 & $-0.32 \pm 0.02$ & 10.0 & $0.12 \pm 0.01$ & 18.0 & $0.059 \pm 0.005$ & 10.90 & 10.86 & 10.87 & 10.85 & $0.47 \pm 0.30$ \\
\hline 35 & PG1149-110 & $0.14 \pm 0.01$ & 11.4 & $0.32 \pm 0.01$ & 17.6 & $0.017 \pm 0.005$ & 10.04 & 10.32 & 10.37 & 10.31 & $0.20 \pm 0.30$ \\
\hline 36 & PG1151+117 & $0.30 \pm 0.11$ & 11.3 & $0.23 \pm 0.07$ & 16.1 & $<0.225$ & 11.11 & 10.99 & 10.99 & 10.85 & $0.39 \pm 0.50$ \\
\hline 37 & PG1202+281 & $0.32 \pm 0.01$ & 11.5 & $0.22 \pm 0.01$ & 17.7 & $0.052 \pm 0.006$ & 11.12 & 11.26 & 11.30 & 11.23 & $1.11 \pm 0.30$ \\
\hline 38 & PG1211+143 & $0.18 \pm 0.01$ & 10.4 & $0.20 \pm 0.00$ & 19.5 & $0.030 \pm 0.003$ & 11.20 & 11.19 & 11.22 & 11.07 & $0.32 \pm 0.50$ \\
\hline 39 & PG1216+069 & $0.14 \pm 0.03$ & 10.2 & $0.20 \pm 0.03$ & 18.2 & $0.049 \pm 0.013$ & 11.66 & 11.54 & 11.51 & 11.32 & $1.84 \pm 0.50$ \\
\hline 40 & PG1226+023 & $0.11 \pm 0.00$ & 10.4 & $0.15 \pm 0.00$ & 19.6 & $<0.004$ & 12.24 & 12.11 & 12. & 12.05 & $2.11 \pm 0.30$ \\
\hline 41 & PG1229+204 & $0.25 \pm 0.02$ & 10.8 & $0.36 \pm 0.01$ & 18.4 & $0.059 \pm 0.010$ & 10.58 & 10.66 & 10.74 & 10.64 & $0.19 \pm 0.30$ \\
\hline 42 & PG1244+026 & $0.04 \pm 0.01$ & 10.6 & $0.16 \pm 0.01$ & 19.3 & $0.052 \pm 0.004$ & 10.03 & 10.24 & 10.28 & 10.24 & $-0.06 \pm 0.30$ \\
\hline 43 & PG1259+593 & $0.37 \pm 0.01$ & 10.0 & $0.17 \pm 0.01$ & 15.9 & $0.049 \pm 0.005$ & 12.15 & 11.78 & 11.74 & 11.59 & $1.51 \pm 0.30$ \\
\hline 44 & PG1302-102 & $0.29 \pm 0.01$ & 10.9 & $0.19 \pm 0.01$ & 16.4 & $<0.016$ & 11.73 & 11.90 & 11.91 & 11.85 & $1.88 \pm 0.30$ \\
\hline 45 & PG1307+085 & $0.34 \pm 0.09$ & 10.1 & $0.40 \pm 0.03$ & 19.2 & $<0.119$ & 11.10 & 11.19 & 11.20 & 11.03 & $0.66 \pm 0.30$ \\
\hline 46 & PG1309+355 & $0.31 \pm 0.09$ & 10.7 & $0.22 \pm 0.02$ & 19.5 & $<0.070$ & 11.47 & 11.48 & 11.53 & 11.42 & $1.04 \pm 0.30$ \\
\hline 47 & PG1310-108 & $0.08 \pm 0.01$ & 10.7 & $0.40 \pm 0.01$ & 18.7 & $0.028 \pm 0.003$ & 9.93 & 10.17 & 10.21 & 9.96 & $-0.41 \pm 0.50$ \\
\hline 48 & PG1322+659 & $0.20 \pm 0.01$ & 11.4 & $0.20 \pm 0.01$ & 17.9 & $0.021 \pm 0.005$ & 11.19 & 11.08 & 11.10 & 11.03 & $1.36 \pm 0.30$ \\
\hline 49 & PG1341+258 & $0.22 \pm 0.02$ & 10.5 & $0.16 \pm 0.01$ & 19.1 & $0.046 \pm 0.008$ & 10.42 & 10.46 & 10.49 & 10.42 & $-0.03 \pm 0.30$ \\
\hline 50 & PG1351+236 & $0.00 \pm 0.03$ & 3.0 & $0.00 \pm 0.03$ & 3.0 & $0.444 \pm 0.008$ & 9.99 & 10.02 & 10.06 & 10.05 & $0.43 \pm 0.30$ \\
\hline 51 & PG1351+640 & $0.58 \pm 0.01$ & 10.0 & $0.20 \pm 0.00$ & 17.0 & $0.063 \pm 0.003$ & 11.06 & 11.31 & 11.42 & 11.39 & $1.32 \pm 0.30$ \\
\hline 52 & PG1352+183 & $0.17 \pm 0.70$ & 9.8 & $0.32 \pm 0.07$ & 16.1 & $<0.969$ & 11.23 & 10.87 & 10.87 & 10.73 & $-0.01 \pm 0.50$ \\
\hline 53 & PG1354+213 & $0.13 \pm 0.01$ & 10.8 & $0.15 \pm 0.03$ & 15.9 & $<0.029$ & 11.39 & 11.44 & 11.45 & 11.41 & $1.58 \pm 0.30$ \\
\hline 54 & PG1402+261 & $0.22 \pm 0.02$ & 10.5 & $0.14 \pm 0.01$ & 16.2 & $0.028 \pm 0.009$ & 11.52 & 11.39 & 11.39 & 11.38 & $1.47 \pm 0.30$ \\
\hline 55 & PG1404+226 & $0.27 \pm 0.02$ & 10.2 & $0.19 \pm 0.02$ & 17.7 & $0.082 \pm 0.008$ & 10.41 & 10.39 & 10.40 & 10.29 & $0.40 \pm 0.30$ \\
\hline 56 & PG1411+442 & $0.16 \pm 0.01$ & 10.2 & $0.11 \pm 0.00$ & 18.0 & $<0.006$ & 11.36 & 10.99 & 10.97 & 10.83 & $0.15 \pm 0.30$ \\
\hline 57 & PG1415+451 & $0.04 \pm 0.01$ & 11.8 & $0.08 \pm 0.01$ & 17.9 & $0.112 \pm 0.004$ & 10.81 & 10.83 & 10.85 & 10.78 & $0.51 \pm 0.30$ \\
\hline 58 & PG1416-129 & $0.55 \pm 0.03$ & 11.8 & $0.49 \pm 0.02$ & 16.7 & $<0.057$ & 10.71 & 10.69 & 10.71 & 10.50 & $0.03 \pm 0.30$ \\
\hline 59 & PG1425+267 & $0.15 \pm 0.01$ & 10.6 & $0.06 \pm 0.01$ & 16.3 & $<0.015$ & 11.78 & 11.84 & 11.85 & 11.82 & $1.78 \pm 0.30$ \\
\hline 60 & PG1426+015 & $0.23 \pm 0.01$ & 10.6 & $0.22 \pm 0.00$ & 17.1 & $0.035 \pm 0.004$ & 11.08 & 11.10 & 11.11 & 11.00 & $1.06 \pm 0.30$ \\
\hline 61 & PG1427+480 & $0.14 \pm 0.01$ & 11.7 & $0.15 \pm 0.01$ & 16.8 & $<0.021$ & 11.18 & 11.27 & 11.30 & 11.34 & $1.10 \pm 0.30$ \\
\hline 62 & PG1435-067 & $0.21 \pm 0.02$ & 10.5 & $0.17 \pm 0.02$ & 18.2 & $<0.034$ & 10.91 & 10.83 & 10.82 & 10.64 & $0.25 \pm 0.50$ \\
\hline 63 & PG1440+356 & $0.07 \pm 0.00$ & 11.8 & $0.12 \pm 0.00$ & 18.3 & $0.142 \pm 0.005$ & 10.98 & 10.90 & 10.94 & 10.95 & $1.33 \pm 0.30$ \\
\hline 64 & PG1444+407 & $0.23 \pm 0.01$ & 10.2 & $0.07 \pm 0.01$ & 18.6 & $0.040 \pm 0.004$ & 11.62 & 11.64 & 11.65 & 11.57 & $1.50 \pm 0.30$ \\
\hline 65 & PG1448+273 & $0.09 \pm 0.01$ & 11.8 & $0.33 \pm 0.01$ & 17.7 & $0.048 \pm 0.004$ & 10.44 & 10.60 & 10.64 & 10.51 & $0.03 \pm 0.30$ \\
\hline
\end{tabular}


Table 4

(Continued)

\begin{tabular}{|c|c|c|c|c|c|c|c|c|c|c|c|}
\hline ID & Name & $\operatorname{Str}_{9.7 \mu \mathrm{m}-\mathrm{Sil}}$ & $\begin{array}{c}\lambda_{9.7 \mu \mathrm{m}-\mathrm{Sil}}^{\text {peak }} \\
\quad(\mu \mathrm{m})\end{array}$ & $\operatorname{Str}_{18 \mu \mathrm{m}-\mathrm{Sil}}$ & $\begin{array}{c}\lambda_{18 \mu \mathrm{m}-\mathrm{Sil}}^{\text {peak }} \\
(\mu \mathrm{m})\end{array}$ & $\begin{array}{c}\mathrm{EW}_{11.3 \mu \mathrm{mPAH}} \\
(\mu \mathrm{m})\end{array}$ & $\begin{array}{c}\log L_{5 \mu \mathrm{m}} \\
\left(\log \left(L_{\odot}\right)\right)\end{array}$ & $\begin{array}{l}\log L_{15 \mu \mathrm{m}} \\
\left(\log \left(L_{\odot}\right)\right)\end{array}$ & $\begin{array}{l}\log L_{18 \mu \mathrm{m}} \\
\left(\log \left(L_{\odot}\right)\right)\end{array}$ & $\begin{array}{l}\log L_{25 \mu \mathrm{m}} \\
\left(\log \left(L_{\odot}\right)\right)\end{array}$ & $\begin{array}{c}\log (\mathrm{SFR}) \\
\left(M_{\odot} \mathrm{yr}^{-1}\right)\end{array}$ \\
\hline 66 & PG1501+106 & $0.00 \pm 0.01$ & 3.0 & $0.25 \pm 0.00$ & 18.5 & $<0.008$ & 10.44 & 10.65 & 10.68 & 10.58 & $-0.63 \pm 0.30$ \\
\hline 67 & PG1512+370 & $.39 \pm 0.01$ & 10.6 & $0.13 \pm 0.01$ & 19.5 & $<0.018$ & 11.70 & 11.70 & 11.70 & 11.63 & $1.66 \pm 0.30$ \\
\hline 68 & PG1519+226 & $.05 \pm 0.01$ & 10.4 & $0.06 \pm 0.01$ & 17.5 & $0.025 \pm 0.005$ & 11.13 & 11.04 & 11.03 & 10.95 & $0.70 \pm 0.30$ \\
\hline 69 & PG1534+580 & $.12 \pm 0.00$ & 11.1 & $0.33 \pm 0.00$ & 18.0 & $0.020 \pm 0.002$ & 9.97 & 10.12 & 10.16 & 10.02 & $-0.82 \pm 0.30$ \\
\hline 70 & PG1535+547 & $0.12 \pm 0.01$ & 10.6 & $0.19 \pm 0.01$ & 18.4 & $0.015 \pm 0.003$ & 10.29 & 10.07 & 10.05 & 9.86 & $-0.65 \pm 0.30$ \\
\hline 71 & PG1543+489 & $0.26 \pm 0.01$ & 11.8 & $0.13 \pm 0.01$ & 15.6 & $<0.007$ & 12.03 & 12.27 & 12.27 & 12.32 & $2.46 \pm 0.30$ \\
\hline 72 & PG1545+210 & $0.28 \pm 0.04$ & 11.6 & $0.34 \pm 0.03$ & 19.1 & $<0.066$ & 11.52 & 11.39 & 11.41 & 11.20 & $1.12 \pm 0.30$ \\
\hline 73 & PG1552+085 & $0.24 \pm 0.01$ & 10.4 & $0.16 \pm 0.02$ & 15.6 & $0.035 \pm 0.007$ & 10.74 & 10.64 & 10.60 & 10.48 & $-0.04 \pm 0.50$ \\
\hline 74 & PG1612+261 & $0.04 \pm 0.01$ & 11.8 & $0.24 \pm 0.01$ & 17.1 & $0.041 \pm 0.003$ & 11.01 & 11.15 & 11.19 & 11.16 & $1.02 \pm 0.30$ \\
\hline 75 & PG1613+658 & $0.24 \pm 0.01$ & 10.4 & $0.24 \pm 0.00$ & 17.5 & $0.054 \pm 0.004$ & 11.59 & 11.58 & 11.59 & 11.55 & $2.02 \pm 0.30$ \\
\hline 76 & PG1617+175 & $0.26 \pm 0.02$ & 10.1 & $0.11 \pm 0.01$ & 18.9 & $<0.039$ & & & & & $0.13 \pm 0.50$ \\
\hline 77 & PG1626+554 & $0.44 \pm 0.02$ & 10.3 & $0.28 \pm 0.01$ & 19.6 & & & & & & $-0.09 \pm 0.50$ \\
\hline 78 & PG1700+518 & & 10.6 & $0.09 \pm 0.01$ & & $0.071 \pm 0.008$ & & & & & $2.07 \pm 0.30$ \\
\hline 79 & PG1704+608 & $0.39 \pm 0.01$ & 10.7 & $0.22 \pm 0.01$ & 17.3 & $<0.024$ & 12.14 & 12.14 & 12.22 & 12.22 & $2.08 \pm 0.30$ \\
\hline 80 & PG2112+059 & $.26 \pm 0.01$ & 10.2 & $0.01 \pm 0.01$ & 18.6 & $0.056 \pm 0.004$ & 12.41 & 12.29 & 12.28 & 12.21 & $2.08 \pm 0.30$ \\
\hline 81 & PG2130+099 & $.07 \pm 0.00$ & 11.9 & $0.15 \pm 0.01$ & 16.4 & $0.012 \pm 0.003$ & & 10.99 & 10.98 & 10.92 & $0.46 \pm 0.30$ \\
\hline 82 & PG2209+184 & $0.27 \pm 0.02$ & 10.4 & $0.27 \pm 0.09$ & 17.6 & $0.146 \pm 0.010$ & 10.29 & 10.12 & 10.11 & 9.91 & $0.45 \pm 0.30$ \\
\hline 83 & PG2214+139 & $0.26 \pm 0.01$ & 10.2 & $0.18 \pm 0.01$ & 18.2 & $0.020 \pm 0.005$ & 10.93 & 10.66 & 10.66 & 10.46 & $-0.01 \pm 0.30$ \\
\hline 84 & PG2233+134 & $0.20 \pm 0.03$ & 11.3 & $0.20 \pm 0.02$ & 19.4 & $<0.049$ & 11.65 & 11.75 & 11.82 & 11.74 & $1.78 \pm 0.30$ \\
\hline 85 & PG2251+113 & $0.32 \pm 0.02$ & 11.0 & $0.35 \pm 0.02$ & 17.7 & $<0.027$ & 11.79 & 11.71 & 11.70 & 11.54 & $1.67 \pm 0.30$ \\
\hline 86 & PG2304+042 & $0.40 \pm 0.01$ & 10.6 & $0.57 \pm 0.01$ & 17.1 & $<0.032$ & 9.70 & 9.75 & 9.75 & 9.45 & $<-0.81$ \\
\hline 87 & PG2308+098 & $0.37 \pm 0.02$ & 10.8 & $0.21 \pm 0.03$ & 18.8 & $<0.033$ & 11.88 & 11.74 & 11.73 & 11.59 & $<1.66$ \\
\hline 88 & 2MASSiJ000703.6+155423 & $0.00 \pm 0.07$ & 3.0 & $0.00 \pm 0.07$ & 3.0 & $0.062 \pm 0.015$ & 10.71 & 10.83 & 10.85 & 10.93 & $1.24 \pm 0.30$ \\
\hline 89 & 2MASSiJ000810.8+135452 & & & & & & & & & & $1.05 \pm 0.30$ \\
\hline 90 & 2MASSiJ004118.7+281640 & $0.41 \pm 0.07$ & 10.7 & $0.17 \pm 0.08$ & 15.7 & $<0.127$ & 11.53 & 11.45 & & 11.40 & $1.45 \pm 0.30$ \\
\hline 91 & 2MASSiJ005010.1+280619 & $0.00 \pm 0.57$ & 3.0 & $0.00 \pm 0.57$ & 3.0 & $2.357 \pm 0.141$ & 10.66 & & & 10.99 & $1.90 \pm 0.30$ \\
\hline 92 & 2MASSiJ005055.7+293328 & $-0.33 \pm 0.04$ & 10.0 & $-0.12 \pm 0.02$ & 19.6 & $<0.036$ & 10.95 & 10.98 & 10.97 & & $1.20 \pm 0.30$ \\
\hline 93 & 2MASSiJ010230.1+262337 & $-0.18 \pm 0.21$ & 9.9 & $-0.15 \pm 0.17$ & 16.4 & $<0.153$ & 10.97 & 10.81 & 10. & 10. & $0.46 \pm 0.50$ \\
\hline 94 & 2MASSiJ010835.1+214818 & $-0.17 \pm 0.03$ & 9.6 & $0.07 \pm 0.01$ & 19.0 & $0.039 \pm 0.007$ & 11.70 & 11.79 & 11.80 & 11.78 & $1.71 \pm 0.30$ \\
\hline 95 & 2MASSiJ012031.5+200327 & $-0.89 \pm 1.07$ & 10.0 & $-0.69 \pm 0.50$ & 16.1 & $0.693 \pm 0.188$ & 9.87 & 9.43 & 9.57 & 9.75 & $0.37 \pm 0.50$ \\
\hline 96 & 2MASSiJ015721.0+171248 & $-0.74 \pm 0.18$ & 10.2 & $-0.36 \pm 0.08$ & 16.5 & $0.083 \pm 0.021$ & 11.24 & 11.08 & 11.10 & 11.25 & $1.77 \pm 0.30$ \\
\hline 97 & 2MASSiJ022150.6+132741 & $0.04 \pm 0.02$ & 11.9 & $0.07 \pm 0.02$ & 15.5 & $<0.071$ & 11.09 & 11.20 & 11.22 & 11.34 & $1.61 \pm 0.30$ \\
\hline 98 & 2MASSiJ024807.3+145957 & $0.04 \pm 0.11$ & 11.2 & $-0.08 \pm 0.10$ & 18.6 & $<0.109$ & 10.48 & 10.55 & 10.52 & 10.53 & $0.49 \pm 0.50$ \\
\hline 99 & 2MASSiJ034857.6+125547 & $-0.50 \pm 0.03$ & 10.1 & $-0.27 \pm 0.01$ & 16.8 & $<0.013$ & 12.00 & 11.84 & 11.79 & 11.85 & $1.84 \pm 0.30$ \\
\hline 100 & 2MASSiJ081652.2+425829 & $0.39 \pm 0.12$ & 10.4 & $0.16 \pm 0.08$ & 19.6 & $<0.139$ & 11.01 & & 10.82 & 10.64 & $<0.94$ \\
\hline 101 & 2MASSiJ082311.3+435318 & $0.14 \pm 0.09$ & 11.8 & $0.19 \pm 0.07$ & 16.2 & & & & & 11. & $1.67 \pm 0.30$ \\
\hline 102 & 2MASSiJ091848.6+211717 & $-0.13 \pm 0.03$ & 11.2 & $0.06 \pm 0.04$ & 15 . & $0.099 \pm 0.013$ & 11.1 & & & & $1.38 \pm 0.30$ \\
\hline 103 & 2MASSiJ092049.0+190320 & $-0.70 \pm 0.41$ & 9.9 & $-0.38 \pm 0.16$ & 16.2 & $<0.199$ & 10. & & & 10. & \\
\hline 104 & 2MASSiJ094636.4+20 & $20 \pm 0.16$ & 10.4 & $0.19 \pm 0.12$ & 19.6 & $<0$. & 11.63 & 11. & 11. & 5 & $1.37 \pm 0.30$ \\
\hline 105 & 2MASSiJ095504.5+170556 & $0.18 \pm 0.09$ & 10.2 & $0.28 \pm 0.05$ & 19.5 & $<0.100$ & 10.75 & 10.60 & 10.59 & 10.41 & $0.09 \pm 0.50$ \\
\hline 106 & 2MASSiJ100121.1+215011 & $-0.95 \pm 0.94$ & 10.3 & $-0.50 \pm 0.16$ & 16.7 & $0.434 \pm 0.062$ & 11.24 & 11.03 & 10.99 & 11.29 & $1.88 \pm 0.30$ \\
\hline 107 & 2MASSiJ101400.4+194614 & & & & & & & & & & $1.08 \pm 0.30$ \\
\hline 108 & 2MASSiJ101649.3+215435 & $0.19 \pm 0.11$ & 10.4 & $0.14 \pm 0.07$ & 20.5 & $<0.128$ & 11.15 & 11.11 & 11.19 & 11.10 & $1.14 \pm 0.30$ \\
\hline 109 & 2MASSiJ102724.9+121920 & $-0.24 \pm 0.07$ & 9.8 & $-0.05 \pm 0.03$ & 16.4 & $0.043 \pm 0.014$ & 11.61 & 11.61 & 11.63 & 11.64 & $1.48 \pm 0.30$ \\
\hline 110 & 2MASSiJ105144.2+353930 & $-0.36 \pm 0.07$ & 10.0 & $0.12 \pm 0.03$ & 16.9 & $<0.062$ & 10.73 & 10.88 & 10.90 & 10.89 & $1.01 \pm 0.30$ \\
\hline 111 & 2MASSiJ125807.4+232921 & $-0.35 \pm 0.03$ & 10.1 & $-0.13 \pm 0.01$ & 16.5 & $<0.019$ & 11.61 & 11.69 & 11.67 & 11.67 & $1.63 \pm 0.30$ \\
\hline 112 & 2MASSiJ130005.3+163214 & $-0.21 \pm 0.03$ & 9.9 & $-0.05 \pm 0.02$ & 15.2 & $<0.030$ & 11.02 & 11.01 & 10.99 & 10.83 & $-0.04 \pm 0.50$ \\
\hline 113 & 2MASSiJ130700.6+233805 & $-1.59 \pm 0.06$ & 10.4 & $-0.54 \pm 0.01$ & 17.1 & $0.032 \pm 0.005$ & 11.95 & 11. & 11.70 & 12.0 & $2.37 \pm 0.30$ \\
\hline 114 & 2MASSiJ132917.5+121340 & $0.11 \pm 0.16$ & 11.1 & $-0.12 \pm 0.19$ & 16.4 & $<0.199$ & 10.78 & 10.76 & 10.72 & 10.64 & $<1.02$ \\
\hline 115 & 2MASSiJ134915.2+220032 & & & & & & & & & & $0.22 \pm 0.30$ \\
\hline 116 & 2MASSiJ1402511+263117 & $0.10 \pm 0.04$ & 11.8 & $0.12 \pm 0.04$ & 19.0 & $<0.068$ & 11.20 & 11.01 & 10.99 & 10.79 & $0.38 \pm 0.50$ \\
\hline 117 & 2MASSiJ145331.5+135358 & $-1.78 \pm 0.07$ & 10.1 & $-0.37 \pm 0.01$ & 17.9 & $0.041 \pm 0.005$ & 11.25 & 11.21 & 11.19 & 11.44 & $1.82 \pm 0.30$ \\
\hline 118 & 2MASSiJ145406.6+195028 & $-0.43 \pm 0.19$ & 10.5 & $-0.37 \pm 0.13$ & 16.7 & $0.169 \pm 0.052$ & 11.14 & 10.98 & 11.01 & 11.07 & $1.08 \pm 0.30$ \\
\hline 119 & 2MASSiJ145410.1+195648 & $-0.26 \pm 0.25$ & 9.9 & $-0.12 \pm 0.13$ & 16.4 & $<0.132$ & 11.47 & 11.28 & 11.27 & 11.18 & $1.22 \pm 0.30$ \\
\hline 120 & 2MASSiJ145608.6+275008 & $0.14 \pm 0.09$ & 10.9 & $0.07 \pm 0.07$ & 19.0 & $<0.160$ & 11.41 & 11.26 & 11.28 & 11.21 & $1.64 \pm 0.30$ \\
\hline 121 & 2MASSiJ150113.1+232908 & $0.14 \pm 0.03$ & 11.9 & $0.07 \pm 0.01$ & 18.4 & $<0.042$ & 11.41 & 11.45 & 11.53 & 11.62 & $1.64 \pm 0.30$ \\
\hline 122 & 2MASSiJ151621.1+225944 & $-0.09 \pm 0.14$ & 11.2 & $-0.39 \pm 0.22$ & 16.5 & $0.208 \pm 0.068$ & 11.06 & 10.81 & 10.81 & 10.79 & $0.68 \pm 0.50$ \\
\hline 123 & 2MASSiJ151653.2+190048 & $0.25 \pm 0.02$ & 10.1 & $0.10 \pm 0.01$ & 19.0 & $<0.032$ & 11.89 & 11.68 & 11.67 & 11.55 & $1.23 \pm 0.30$ \\
\hline 124 & 2MASSiJ151901.5+183804 & $0.37 \pm 0.07$ & 11.7 & $-0.11 \pm 0.09$ & 15.7 & $<0.213$ & 10.75 & 10.46 & 10.51 & 10.50 & $0.25 \pm 0.50$ \\
\hline 125 & 2MASSiJ152151.0+225120 & $-0.25 \pm 0.20$ & 10.0 & $-0.28 \pm 0.11$ & 16.5 & & & & & & $2.46 \pm 0.30$ \\
\hline 126 & 2MASSiJ154307.7+193751 & $-0.12 \pm 0.08$ & 12.1 & $0.08 \pm 0.05$ & 20.4 & $0.094 \pm 0.024$ & 11.71 & 11.64 & 11.64 & 11.54 & $1.46 \pm 0.30$ \\
\hline 127 & 2MASSiJ163700.2+222114 & $-0.11 \pm 0.07$ & 9.8 & $0.08 \pm 0.03$ & 18.7 & $0.205 \pm 0.021$ & 10.67 & 10.68 & 10.64 & 10.69 & $1.54 \pm 0.30$ \\
\hline 128 & 2MASSiJ163736.5+254302 & $-0.39 \pm 0.21$ & 10.0 & $-0.51 \pm 0.20$ & 16.5 & $<0.182$ & 11.38 & 11.44 & 11.49 & 11.51 & $1.51 \pm 0.30$ \\
\hline 129 & 2MASSiJ165939.7+183436 & $-0.23 \pm 0.02$ & 10.6 & $-0.16 \pm 0.01$ & 16.4 & $0.027 \pm 0.004$ & 11.32 & 11.53 & 11.54 & 11.54 & $1.60 \pm 0.30$ \\
\hline 130 & 2MASSiJ170536.6+210137 & $0.10 \pm 0.12$ & 12.4 & $-0.38 \pm 0.18$ & 15.5 & $0.486 \pm 0.063$ & 10.77 & 10.73 & 10.92 & 11.02 & $1.53 \pm 0.30$ \\
\hline
\end{tabular}


Table 4

(Continued)

\begin{tabular}{|c|c|c|c|c|c|c|c|c|c|c|c|}
\hline ID & Name & $\operatorname{Str}_{9.7 \mu \mathrm{m}-\mathrm{Sil}}$ & $\begin{array}{l}\lambda_{9.7 \mu \mathrm{m}-\mathrm{Sil}}^{\text {peak }} \\
\quad(\mu \mathrm{m})\end{array}$ & $\operatorname{Str}_{18 \mu \mathrm{m}-\mathrm{Sil}}$ & $\begin{array}{c}\lambda_{18 \mu \mathrm{peak}-\mathrm{Sil}}^{\text {peal }} \\
\quad(\mu \mathrm{m})\end{array}$ & $\begin{array}{c}\mathrm{EW}_{11.3 \mu \mathrm{mPAH}} \\
(\mu \mathrm{m})\end{array}$ & $\begin{array}{l}\log L_{5 \mu \mathrm{m}} \\
\left(\log \left(L_{\odot}\right)\right)\end{array}$ & $\begin{array}{l}\log L_{15 \mu \mathrm{m}} \\
\left(\log \left(L_{\odot}\right)\right)\end{array}$ & $\begin{array}{l}\log L_{18 \mu \mathrm{m}} \\
\left(\log \left(L_{\odot}\right)\right)\end{array}$ & $\begin{array}{l}\log L_{25} \mu \mathrm{m} \\
\left(\log \left(L_{\odot}\right)\right)\end{array}$ & $\begin{array}{c}\log (\mathrm{SFR}) \\
\left(M_{\odot} \mathrm{yr}^{-1}\right)\end{array}$ \\
\hline 131 & 2MASSiJ171442.7+260248 & $0.02 \pm 0.03$ & 11.6 & $-0.30 \pm 0.04$ & 19.6 & $<0.053$ & 11.09 & 10.98 & 10.88 & 10.79 & $0.64 \pm 0.50$ \\
\hline 132 & 2MASSiJ222221.1+195947 & $0.19 \pm 0.02$ & 11.2 & $0.15 \pm 0.02$ & 19.0 & $<0.038$ & 11.40 & 11.35 & 11.35 & 11.21 & $1.29 \pm 0.30$ \\
\hline 133 & 2MASSiJ222554.2+195837 & $-0.61 \pm 0.05$ & 10.0 & $-0.08 \pm 0.02$ & 18.7 & $<0.037$ & 10.86 & 10.94 & 10.90 & 10.95 & $1.33 \pm 0.30$ \\
\hline 134 & 2MASSiJ223742.6+145614 & $0.19 \pm 0.10$ & 10.7 & $-0.21 \pm 0.11$ & 16.3 & $0.229 \pm 0.049$ & 11.22 & 11.13 & 11.20 & 11.14 & $1.64 \pm 0.30$ \\
\hline 135 & 2MASSiJ223946.0+192955 & $0.37 \pm 0.14$ & 12.3 & $-0.44 \pm 0.30$ & 16.3 & $1.134 \pm 0.287$ & 10.57 & 10.31 & 10.42 & 10.52 & $1.24 \pm 0.30$ \\
\hline 136 & 2MASSiJ230304.3+162440 & $-0.90 \pm 0.60$ & 10.0 & $-1.16 \pm 0.29$ & 17.5 & $1.186 \pm 0.082$ & 10.20 & 10.26 & 10.69 & 10.72 & $1.71 \pm 0.30$ \\
\hline 137 & 2MASSiJ230442.4+270616 & $0.29 \pm 0.19$ & 10.6 & $0.29 \pm 0.08$ & 19.6 & $<0.338$ & 10.37 & 10.35 & 10.47 & 10.50 & $0.63 \pm 0.50$ \\
\hline 138 & 2MASSiJ234259.3+134750 & $0.29 \pm 0.15$ & 10.1 & $-0.25 \pm 0.12$ & 15.1 & $<0.161$ & 11.10 & 11.11 & 11.25 & 11.21 & $1.32 \pm 0.30$ \\
\hline 139 & 2MASSiJ234449.5+122143 & $0.10 \pm 0.04$ & 11.2 & $0.04 \pm 0.02$ & 20.5 & $<0.054$ & 11.26 & 11.29 & 11.32 & 11.33 & $1.53 \pm 0.30$ \\
\hline
\end{tabular}
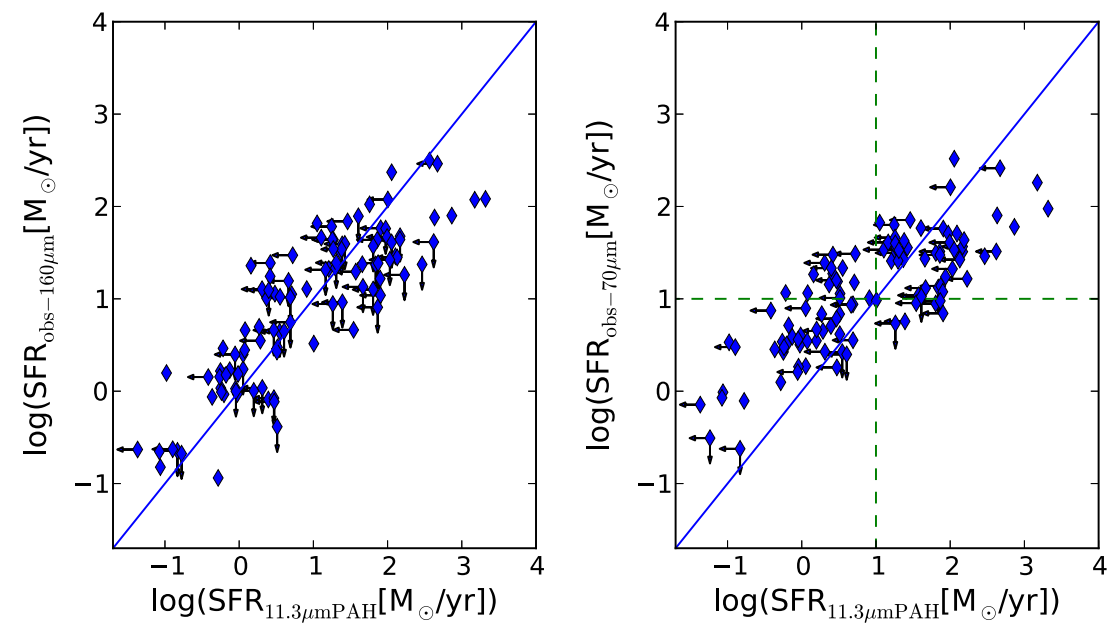

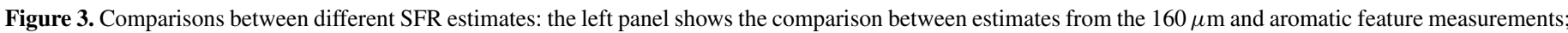
the right panel shows the comparison between $70 \mu \mathrm{m}$ based and aromatic-feature-based SFR estimates.

(A color version of this figure is available in the online journal.)

based on the scatter of Figure 3. Figure 1 shows the best-fit starforming template for each quasar. The residual after subtracting the star-forming template is adopted as the radiation from the dusty torus.

\section{RESULTS}

\subsection{Composite Quasar SEDs and Scatter about Them}

Figure 4 shows the median composite spectra with $1 \sigma$ scatter for the PG and 2MASS samples normalized at 14-16 $\mu \mathrm{m}$. The composite spectrum from 5 to $25 \mu \mathrm{m}$ is derived by taking the median of the spectroscopic data projected to a common wavelength grid with a spectral resolution of 50 . We took the median of all the $70 \mu \mathrm{m}$ photometry with the rest-frame wavelength from 40 to $70 \mu \mathrm{m}$ as the composite flux density at $55 \mu \mathrm{m}$, and that of $160 \mu \mathrm{m}$ data with the rest-frame wavelength from 100 to $160 \mu \mathrm{m}$ as the composite flux density at $130 \mu \mathrm{m}$. We also constructed composite spectra for the radio-quite and radio-loud sub-samples of $\mathrm{PG}$ objects, where the radio-loudness data are from Kellermann et al. (1989), as indicated in Table 5. All the PG objects have mid-IR spectroscopic measurements, while $90 \%$ and $76 \%$ of the sample have detections at 70 and $160 \mu \mathrm{m}$, respectively. Ninety-five percent of the 2MASS sample have mid-IR spectroscopic measurements and $70 \mu$ m detections, with $160 \mu \mathrm{m}$ detections for $33 \%$. The median spectra presented in Figure 4 should be representative of the whole PG sample up to rest-frame $130 \mu \mathrm{m}$ and of the 2MASS sample to rest-frame $60 \mu \mathrm{m}$, while the rest-frame $130 \mu \mathrm{m}$ data point of the $2 \mathrm{MASS}$ median spectrum should be biased by the far-IR luminous objects in the sample. There are some differences in the median spectra between the 2MASS and PG samples. While the PG median spectrum shows silicate emission, the 2MASS one has silicate absorption, consistent with the near-IR selected 2MASS quasars being viewed on average more edge-on. In addition, the 2MASS spectrum has much larger equivalent widths (EWs) of the aromatic features compared to the PG spectrum, as well as elevated rest-frame $60 \mu \mathrm{m}$ emission relative to the mid-IR. This behavior indicates more active SF relative to the nuclear SMBH luminosity in 2MASS quasar host galaxies (Shi et al. 2007).

Figure 3 compares the SEDs we have determined with a number of alternatives. Elvis et al. (1994) presented composite SEDs of 47 non-blazar quasars, largely based on members of the PG sample. Their IR data are based on ground-based $N$ and $Q$ bands $(10$ and $20 \mu \mathrm{m})$ as well as IRAS broad-band photometry $(12,25,60$, and $100 \mu \mathrm{m})$. As shown in Figure 4, their radioquiet composite SED shows an overall similar shape to that of radio-quiet PG quasars from our work below rest-frame $60 \mu \mathrm{m}$ but is significantly lower at rest-frame $130 \mu \mathrm{m}$. However, their data do not include photometry at wavelengths longer than observed-frame $100 \mu \mathrm{m}$ and the SED at longer wavelengths is based on extrapolation. On the other hand, their radio-loud quasar composite SED is similar to ours between rest-frame 5 and $130 \mu \mathrm{m}$. Richards et al. (2006) compiled Spitzer broad-band photometry of 259 Sloan-Digital-Sky-Survey-selected optically bright quasars at $3.6,4.5,5.8,8.0,24$, and $70 \mu \mathrm{m}$. Their sample covers a redshift range from $z=0$ up to $z=4$. 
Table 5

The Median Composite Spectra of PG and 2MASS Samples

\begin{tabular}{|c|c|c|c|c|}
\hline Wave $(\mu \mathrm{m})$ & $\begin{array}{c}\log \left(v L_{v}\right) \\
\text { All PG }\end{array}$ & $\begin{array}{c}\log \left(v L_{v}\right) \\
\text { Radio-quite PG }\end{array}$ & $\begin{array}{c}\log \left(v L_{v}\right) \\
\text { Radio-loud PG }\end{array}$ & $\begin{array}{c}\log \left(v L_{v}\right) \\
\text { All 2MASS }\end{array}$ \\
\hline 5.05 & $0.035 \pm 0.151$ & $0.049 \pm 0.165$ & $0.013 \pm 0.096$ & $0.024 \pm 0.206$ \\
\hline 5.15 & $0.021 \pm 0.156$ & $0.032 \pm 0.171$ & $-0.006 \pm 0.097$ & $0.014 \pm 0.191$ \\
\hline 5.25 & $0.023 \pm 0.161$ & $0.024 \pm 0.174$ & $0.023 \pm 0.096$ & $0.008 \pm 0.246$ \\
\hline 5.36 & $-0.002 \pm 0.173$ & $-0.002 \pm 0.186$ & $0.001 \pm 0.102$ & $0.006 \pm 0.209$ \\
\hline 5.47 & $0.001 \pm 0.159$ & $-0.000 \pm 0.169$ & $0.004 \pm 0.101$ & $0.020 \pm 0.229$ \\
\hline 5.58 & $-0.003 \pm 0.164$ & $-0.006 \pm 0.176$ & $-0.001 \pm 0.099$ & $0.029 \pm 0.191$ \\
\hline 5.69 & $-0.005 \pm 0.150$ & $-0.019 \pm 0.160$ & $0.009 \pm 0.098$ & $0.025 \pm 0.150$ \\
\hline 5.80 & $-0.010 \pm 0.175$ & $-0.017 \pm 0.189$ & $0.011 \pm 0.088$ & $-0.001 \pm 0.201$ \\
\hline 5.92 & $-0.009 \pm 0.154$ & $-0.009 \pm 0.166$ & $0.002 \pm 0.089$ & $0.006 \pm 0.170$ \\
\hline 6.04 & $-0.014 \pm 0.146$ & $-0.017 \pm 0.156$ & $0.013 \pm 0.085$ & $0.018 \pm 0.192$ \\
\hline 6.16 & $-0.006 \pm 0.145$ & $-0.006 \pm 0.156$ & $-0.001 \pm 0.083$ & $0.038 \pm 0.158$ \\
\hline 6.28 & $-0.007 \pm 0.136$ & $-0.008 \pm 0.147$ & $-0.005 \pm 0.080$ & $0.050 \pm 0.167$ \\
\hline 6.40 & $-0.010 \pm 0.135$ & $-0.013 \pm 0.145$ & $-0.004 \pm 0.084$ & $0.022 \pm 0.142$ \\
\hline 6.53 & $-0.026 \pm 0.137$ & $-0.031 \pm 0.148$ & $-0.003 \pm 0.080$ & $-0.007 \pm 0.146$ \\
\hline 6.66 & $-0.027 \pm 0.137$ & $-0.034 \pm 0.147$ & $-0.015 \pm 0.078$ & $-0.013 \pm 0.155$ \\
\hline 6.80 & $-0.025 \pm 0.125$ & $-0.028 \pm 0.135$ & $-0.016 \pm 0.075$ & $-0.008 \pm 0.141$ \\
\hline 6.93 & $-0.022 \pm 0.123$ & $-0.029 \pm 0.133$ & $-0.013 \pm 0.074$ & $0.020 \pm 0.179$ \\
\hline 7.07 & $-0.028 \pm 0.121$ & $-0.034 \pm 0.130$ & $-0.021 \pm 0.074$ & $0.012 \pm 0.152$ \\
\hline 7.21 & $-0.032 \pm 0.119$ & $-0.035 \pm 0.128$ & $-0.022 \pm 0.072$ & $0.019 \pm 0.139$ \\
\hline 7.36 & $-0.032 \pm 0.131$ & $-0.033 \pm 0.144$ & $-0.031 \pm 0.068$ & $0.033 \pm 0.150$ \\
\hline 7.50 & $-0.025 \pm 0.132$ & $-0.029 \pm 0.145$ & $-0.023 \pm 0.071$ & $0.051 \pm 0.184$ \\
\hline 7.65 & $-0.017 \pm 0.119$ & $-0.017 \pm 0.128$ & $-0.017 \pm 0.076$ & $0.072 \pm 0.198$ \\
\hline 7.81 & $-0.031 \pm 0.113$ & $-0.015 \pm 0.122$ & $-0.031 \pm 0.069$ & $0.051 \pm 0.199$ \\
\hline 7.96 & $-0.033 \pm 0.106$ & $-0.023 \pm 0.113$ & $-0.034 \pm 0.069$ & $0.034 \pm 0.176$ \\
\hline 8.12 & $-0.042 \pm 0.105$ & $-0.025 \pm 0.113$ & $-0.045 \pm 0.062$ & $0.041 \pm 0.145$ \\
\hline 8.29 & $-0.049 \pm 0.149$ & $-0.049 \pm 0.165$ & $-0.049 \pm 0.057$ & $0.017 \pm 0.154$ \\
\hline 8.45 & $-0.049 \pm 0.104$ & $-0.048 \pm 0.113$ & $-0.053 \pm 0.053$ & $0.019 \pm 0.149$ \\
\hline 8.62 & $-0.042 \pm 0.111$ & $-0.046 \pm 0.121$ & $-0.040 \pm 0.054$ & $0.025 \pm 0.150$ \\
\hline 8.79 & $-0.034 \pm 0.114$ & $-0.036 \pm 0.124$ & $-0.033 \pm 0.060$ & $-0.010 \pm 0.121$ \\
\hline 8.97 & $-0.035 \pm 0.100$ & $-0.045 \pm 0.109$ & $-0.023 \pm 0.049$ & $-0.027 \pm 0.128$ \\
\hline 9.15 & $-0.020 \pm 0.106$ & $-0.036 \pm 0.116$ & $-0.003 \pm 0.051$ & $-0.042 \pm 0.155$ \\
\hline 9.33 & $-0.014 \pm 0.105$ & $-0.025 \pm 0.114$ & $0.006 \pm 0.052$ & $-0.066 \pm 0.174$ \\
\hline 9.52 & $0.011 \pm 0.103$ & $-0.009 \pm 0.111$ & $0.028 \pm 0.053$ & $-0.066 \pm 0.176$ \\
\hline 9.71 & $0.017 \pm 0.106$ & $-0.002 \pm 0.113$ & $0.049 \pm 0.054$ & $-0.051 \pm 0.200$ \\
\hline 9.90 & $0.024 \pm 0.099$ & $0.009 \pm 0.106$ & $0.042 \pm 0.054$ & $-0.081 \pm 0.222$ \\
\hline 10.10 & $0.023 \pm 0.096$ & $0.019 \pm 0.103$ & $0.062 \pm 0.051$ & $-0.035 \pm 0.170$ \\
\hline 10.30 & $0.026 \pm 0.115$ & $0.020 \pm 0.123$ & $0.068 \pm 0.058$ & $-0.026 \pm 0.154$ \\
\hline 10.51 & $0.047 \pm 0.087$ & $0.033 \pm 0.091$ & $0.088 \pm 0.050$ & $0.003 \pm 0.188$ \\
\hline 10.72 & $0.030 \pm 0.091$ & $0.020 \pm 0.096$ & $0.082 \pm 0.053$ & $-0.012 \pm 0.161$ \\
\hline 10.93 & $0.038 \pm 0.095$ & $0.025 \pm 0.100$ & $0.066 \pm 0.051$ & $0.018 \pm 0.142$ \\
\hline 11.15 & $0.047 \pm 0.084$ & $0.041 \pm 0.090$ & $0.069 \pm 0.048$ & $0.047 \pm 0.155$ \\
\hline 11.37 & $0.048 \pm 0.097$ & $0.048 \pm 0.105$ & $0.055 \pm 0.049$ & $0.046 \pm 0.167$ \\
\hline 11.60 & $0.031 \pm 0.072$ & $0.022 \pm 0.076$ & $0.055 \pm 0.046$ & $0.032 \pm 0.115$ \\
\hline 11.83 & $0.021 \pm 0.076$ & $0.019 \pm 0.082$ & $0.044 \pm 0.038$ & $0.025 \pm 0.099$ \\
\hline 12.07 & $0.023 \pm 0.063$ & $0.018 \pm 0.067$ & $0.032 \pm 0.038$ & $0.030 \pm 0.098$ \\
\hline 12.31 & $0.006 \pm 0.050$ & $0.007 \pm 0.054$ & $0.005 \pm 0.034$ & $0.030 \pm 0.107$ \\
\hline 12.56 & $0.002 \pm 0.047$ & $0.001 \pm 0.051$ & $0.002 \pm 0.029$ & $0.044 \pm 0.113$ \\
\hline 12.81 & $0.005 \pm 0.046$ & $0.006 \pm 0.049$ & $0.001 \pm 0.030$ & $0.050 \pm 0.148$ \\
\hline 13.06 & $-0.011 \pm 0.041$ & $-0.009 \pm 0.044$ & $-0.016 \pm 0.026$ & $0.024 \pm 0.071$ \\
\hline 13.33 & $-0.017 \pm 0.035$ & $-0.012 \pm 0.037$ & $-0.022 \pm 0.022$ & $0.002 \pm 0.064$ \\
\hline 13.59 & $-0.017 \pm 0.030$ & $-0.015 \pm 0.032$ & $-0.020 \pm 0.020$ & $0.005 \pm 0.066$ \\
\hline 13.86 & $-0.020 \pm 0.025$ & $-0.016 \pm 0.026$ & $-0.026 \pm 0.017$ & $-0.004 \pm 0.050$ \\
\hline 14.14 & $-0.014 \pm 0.021$ & $-0.013 \pm 0.020$ & $-0.015 \pm 0.022$ & $0.005 \pm 0.044$ \\
\hline 14.42 & $-0.007 \pm 0.020$ & $-0.007 \pm 0.020$ & $-0.009 \pm 0.019$ & $0.014 \pm 0.059$ \\
\hline 14.71 & $-0.012 \pm 0.018$ & $-0.012 \pm 0.017$ & $-0.010 \pm 0.020$ & $-0.002 \pm 0.047$ \\
\hline 15.01 & $-0.010 \pm 0.016$ & $-0.010 \pm 0.016$ & $-0.007 \pm 0.017$ & $-0.015 \pm 0.047$ \\
\hline 15.31 & $-0.002 \pm 0.020$ & $-0.001 \pm 0.016$ & $-0.004 \pm 0.033$ & $-0.013 \pm 0.059$ \\
\hline 15.61 & $0.024 \pm 0.027$ & $0.024 \pm 0.026$ & $0.028 \pm 0.029$ & $0.014 \pm 0.067$ \\
\hline 15.93 & $0.015 \pm 0.022$ & $0.015 \pm 0.021$ & $0.015 \pm 0.025$ & $-0.011 \pm 0.071$ \\
\hline 16.24 & $0.018 \pm 0.023$ & $0.018 \pm 0.023$ & $0.021 \pm 0.023$ & $-0.005 \pm 0.069$ \\
\hline 16.57 & $0.024 \pm 0.027$ & $0.025 \pm 0.028$ & $0.021 \pm 0.025$ & $0.004 \pm 0.091$ \\
\hline 16.90 & $0.028 \pm 0.031$ & $0.028 \pm 0.031$ & $0.027 \pm 0.030$ & $0.006 \pm 0.097$ \\
\hline 17.24 & $0.024 \pm 0.036$ & $0.025 \pm 0.037$ & $0.024 \pm 0.031$ & $0.009 \pm 0.106$ \\
\hline 17.58 & $0.025 \pm 0.038$ & $0.027 \pm 0.038$ & $0.019 \pm 0.039$ & $0.000 \pm 0.107$ \\
\hline 17.94 & $0.019 \pm 0.036$ & $0.019 \pm 0.035$ & $0.019 \pm 0.037$ & $-0.005 \pm 0.158$ \\
\hline
\end{tabular}


Table 5

(Continued)

\begin{tabular}{|c|c|c|c|c|}
\hline Wave $(\mu \mathrm{m})$ & $\begin{array}{c}\log \left(v L_{v}\right) \\
\text { All PG }\end{array}$ & $\begin{array}{c}\log \left(v L_{v}\right) \\
\text { Radio-quite PG }\end{array}$ & $\begin{array}{c}\log \left(v L_{v}\right) \\
\text { Radio-loud PG }\end{array}$ & $\begin{array}{c}\log \left(v L_{v}\right) \\
\text { All 2MASS }\end{array}$ \\
\hline 18.29 & $0.015 \pm 0.038$ & $0.014 \pm 0.038$ & $0.021 \pm 0.038$ & $-0.003 \pm 0.071$ \\
\hline 18.66 & $0.018 \pm 0.043$ & $0.021 \pm 0.045$ & $0.013 \pm 0.036$ & $0.018 \pm 0.100$ \\
\hline 19.03 & $0.007 \pm 0.043$ & $0.006 \pm 0.044$ & $0.010 \pm 0.036$ & $-0.003 \pm 0.116$ \\
\hline 19.41 & $-0.003 \pm 0.044$ & $-0.003 \pm 0.045$ & $-0.007 \pm 0.038$ & $0.006 \pm 0.084$ \\
\hline 19.80 & $-0.006 \pm 0.046$ & $-0.009 \pm 0.047$ & $-0.001 \pm 0.043$ & $0.012 \pm 0.098$ \\
\hline 20.20 & $-0.016 \pm 0.053$ & $-0.021 \pm 0.053$ & $-0.007 \pm 0.051$ & $0.011 \pm 0.098$ \\
\hline 20.60 & $-0.027 \pm 0.055$ & $-0.031 \pm 0.056$ & $-0.013 \pm 0.052$ & $0.013 \pm 0.117$ \\
\hline 21.01 & $-0.021 \pm 0.061$ & $-0.028 \pm 0.064$ & $-0.014 \pm 0.049$ & $0.014 \pm 0.098$ \\
\hline 21.43 & $-0.028 \pm 0.068$ & $-0.032 \pm 0.069$ & $-0.026 \pm 0.063$ & $0.014 \pm 0.111$ \\
\hline 21.86 & $-0.032 \pm 0.070$ & $-0.036 \pm 0.072$ & $-0.029 \pm 0.061$ & $0.016 \pm 0.121$ \\
\hline 22.30 & $-0.037 \pm 0.074$ & $-0.036 \pm 0.076$ & $-0.045 \pm 0.067$ & $0.010 \pm 0.114$ \\
\hline 22.75 & $-0.046 \pm 0.078$ & $-0.045 \pm 0.081$ & $-0.048 \pm 0.060$ & $0.015 \pm 0.122$ \\
\hline 23.20 & $-0.051 \pm 0.083$ & $-0.050 \pm 0.086$ & $-0.051 \pm 0.069$ & $0.013 \pm 0.134$ \\
\hline 23.66 & $-0.056 \pm 0.098$ & $-0.054 \pm 0.103$ & $-0.066 \pm 0.071$ & $0.011 \pm 0.143$ \\
\hline 24.14 & $-0.059 \pm 0.093$ & $-0.058 \pm 0.097$ & $-0.064 \pm 0.074$ & $0.017 \pm 0.144$ \\
\hline 24.62 & $-0.066 \pm 0.102$ & $-0.066 \pm 0.106$ & $-0.067 \pm 0.081$ & $0.005 \pm 0.154$ \\
\hline 25.11 & $-0.079 \pm 0.105$ & $-0.080 \pm 0.110$ & $-0.065 \pm 0.078$ & $0.001 \pm 0.156$ \\
\hline 55.00 & $-0.301 \pm 0.270$ & $-0.303 \pm 0.276$ & $-0.301 \pm 0.249$ & $-0.090 \pm 0.442$ \\
\hline 130.00 & $-0.611 \pm 0.295$ & $-0.611 \pm 0.290$ & $-0.601 \pm 0.317$ & $-0.251 \pm 0.442$ \\
\hline
\end{tabular}
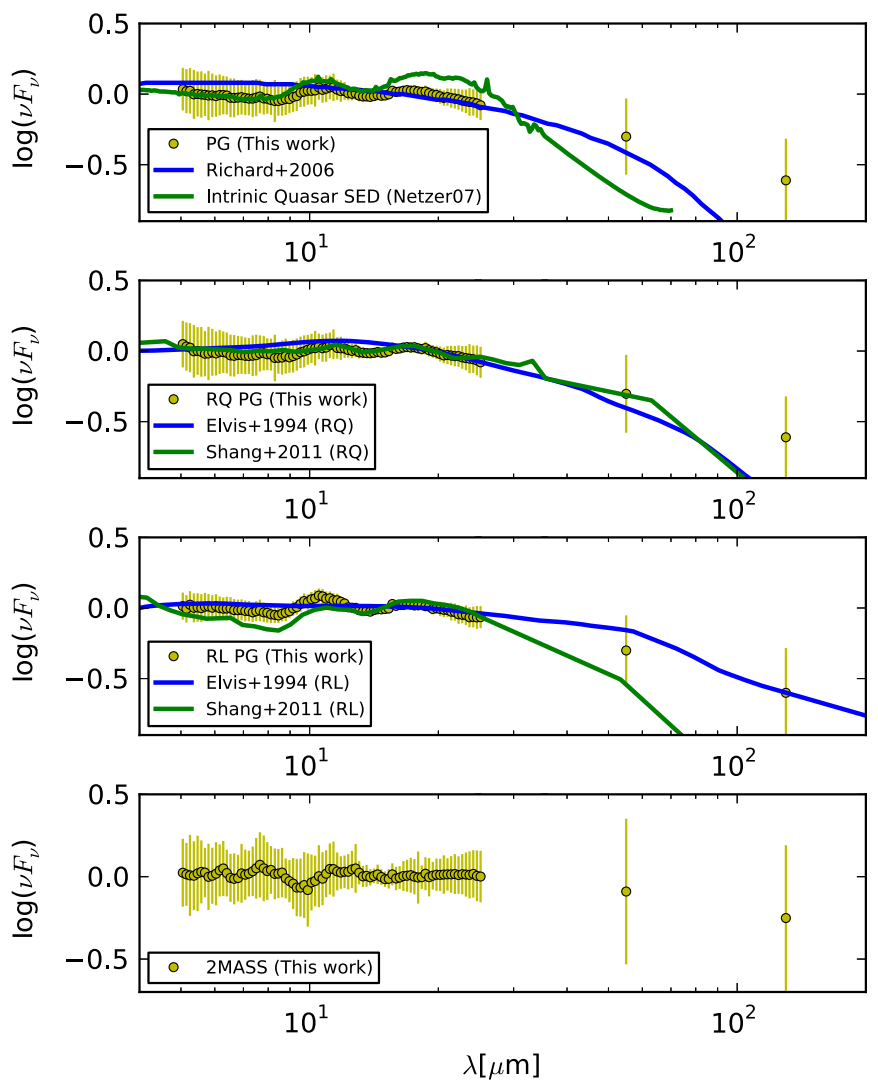

Figure 4. From top to bottom, filled circles indicate composite median SEDs of all PG quasars, radio-quiet PG quasars, radio-loud PG quasars, and all 2MASS quasars, respectively, where the error bar is shown at $1 \sigma$ level. The comparison SEDs include those by Elvis et al. (1994), Richards et al. (2006), Netzer et al. (2007), and Shang et al. (2011).

(A color version of this figure is available in the online journal.)

Compared to our composite spectrum of all PG quasars, their SED shows an overall similar shape between rest-frame 5 and $30 \mu \mathrm{m}$ but lacks silicate emission features. Above restframe $100 \mu \mathrm{m}$, their SED drops faster than ours. Again, their observations do not contain far-IR data above observed $70 \mu \mathrm{m}$ and the shape at longer wavelengths is based on extrapolation.
Table 6

Correlation Between Different IR Color and $15 \mu \mathrm{m}$ Luminosity

\begin{tabular}{lccc}
\hline \hline$Y$ versus $X$ & $A$ & $B$ & $\sigma$ \\
\hline $\log \left(L_{25 \mu \mathrm{m}} / L_{15 \mu \mathrm{m}}\right)$ vs. $\log \left(L_{15 \mu \mathrm{m}}\right)$ & -0.19 & 0.014 & 0.14 \\
$\log \left(L_{25 \mu \mathrm{m}} / L_{5 \mu \mathrm{m}}\right)$ vs. $\log \left(L_{15 \mu \mathrm{m}}\right)$ & -0.37 & 0.028 & 0.23 \\
$\log \left(L_{15 \mu \mathrm{m}} / L_{5} \mu \mathrm{m}\right)$ vs. $\log \left(L_{15 \mu \mathrm{m}}\right)$ & -0.18 & 0.014 & 0.16 \\
\hline
\end{tabular}

Notes. $Y=(A+B X) \pm \sigma$. The monochromatic luminosity is defined to be the average emission over a $2 \mu \mathrm{m}$ width.

Netzer et al. (2007) constructed the intrinsic quasar SED of eight PG quasars after removing the star-forming emission. Their SED resembles our composite spectrum for the whole PG sample up to $30 \mu \mathrm{m}$ above which theirs drops much faster, which is expected as the star-forming radiation starts to dominate the IR emission above $30 \mu \mathrm{m}$. Shang et al. (2011) constructed a composite SED of 85 optically bright, non-blazar quasars that are selected heterogeneously. Their radio-quiet composite SED is quite similar to our radio-quiet PG spectrum between restframe 5 and $30 \mu \mathrm{m}$ but drops faster longward of rest-frame $55 \mu \mathrm{m}$, which is also the case when comparing their radio-loud composite SED to our radio-loud PG one. The comparison of $160 \mu \mathrm{m}$ photometry for about 20 objects in common reveals that their measurements are systematically lower than ours by a factor of a few. Since our independent measurements at this wavelength from Spitzer and Herschel agree much more closely than this factor, we believe our measurements are reliable.

To illustrate the scatter of the mid-IR spectra among the whole PG and 2MASS samples, we present the relationships between IR color as a function of $15 \mu \mathrm{m}$ monochromatic luminosities in Figure 5 and list the best-fit linear relations in Table 6. Almost zero slopes for all of these relationships indicate that the mid-IR spectral shape of PG and 2MASS quasars are roughly independent of quasar mid-IR luminosities. In addition, the scatter of the relationship is quite small, ranging from 0.14 to $0.23 \mathrm{dex}$. This suggests that the composite mid-IR spectra derived above are good representatives of quasar midIR spectra independent of quasar IR luminosities and quasar types. However, these spectra include combinations of SMBHand SF-generated components, as would also be the case for 

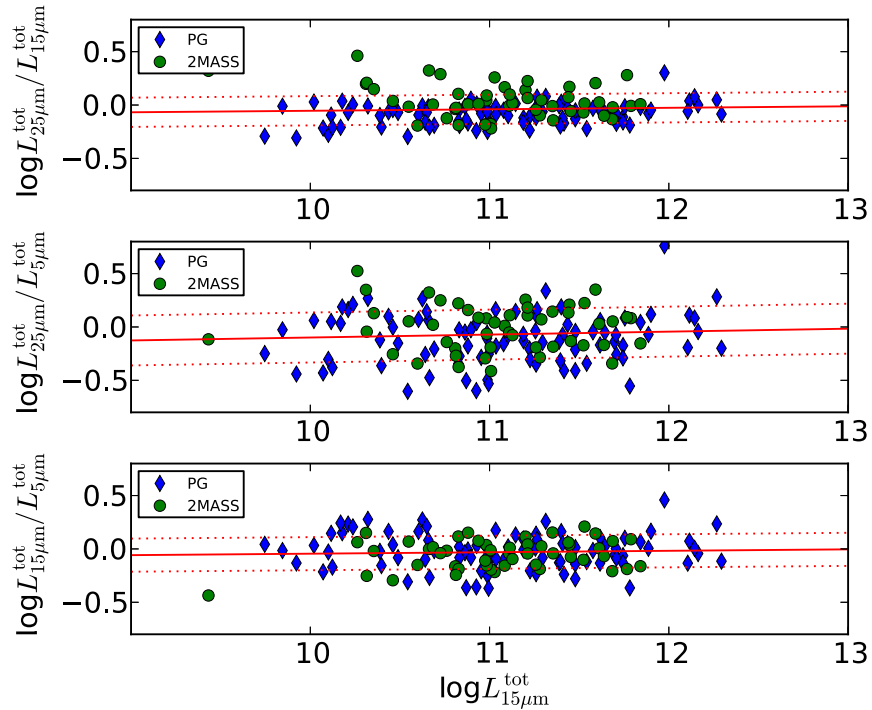

Figure 5. Mid-IR color of $25 \mu \mathrm{m} / 15 \mu \mathrm{m}, 25 \mu \mathrm{m} / 5 \mu \mathrm{m}$, and $15 \mu \mathrm{m} / 5 \mu \mathrm{m}$ as a function of the $15 \mu \mathrm{m}$ luminosity. All luminosities are monochromatic emission averaged over $2 \mu \mathrm{m}$ width of the central wavelength. The best linear fits are shown as solid lines with $1 \sigma$ scatter as the dotted line. The fitting results are also listed in Table 6.

(A color version of this figure is available in the online journal.)

the previously determined quasar spectral templates (e.g., Elvis et al. 1994; Richards et al. 2006; Shang et al. 2011). Obtaining an intrinsic quasar SED template in the far-IR requires subtracting the SF contribution.

\subsection{Contribution of Star Formation to the SED}

The left panel of Figure 6 shows the IR color $\log \left(L_{60 \mu \mathrm{m}} / L_{25 \mu \mathrm{m}}\right)$ as a function of the EW of the $11.3 \mu \mathrm{m}$ aromatic feature. This color varies from -0.8 to 0.8 for the whole quasar sample, but it clusters in a smaller range from -0.5 to 0.25 . The $\mathrm{EW}_{11.3 \mu \mathrm{mPAH}}$ for the sample ranges from $<0.003 \mu \mathrm{m}$ to $2.3 \mu \mathrm{m}$. As shown in the figure, the two quantities are roughly related to each other, with increasing $\log \left(L_{60 \mu \mathrm{m}} / L_{25 \mu \mathrm{m}}\right)$ at increasing $\mathrm{EW}_{11.3 \mu \mathrm{mPAH}}$, which indicates that the rest-frame IR color $\log \left(L_{60 \mu \mathrm{m}} / L_{25 \mu \mathrm{m}}\right)$ is to the first order driven by the relative brightness of star-forming regions and the dusty tori. A relationship between IR color $f_{30 \mu \mathrm{m}} / L_{15 \mu \mathrm{m}}$ and $\mathrm{EW}_{7.7} \mu \mathrm{mPAH}$ is also observed in the sample of Veilleux et al. (2009) that is composed of PG quasars and ULIRGs. However, such correla-
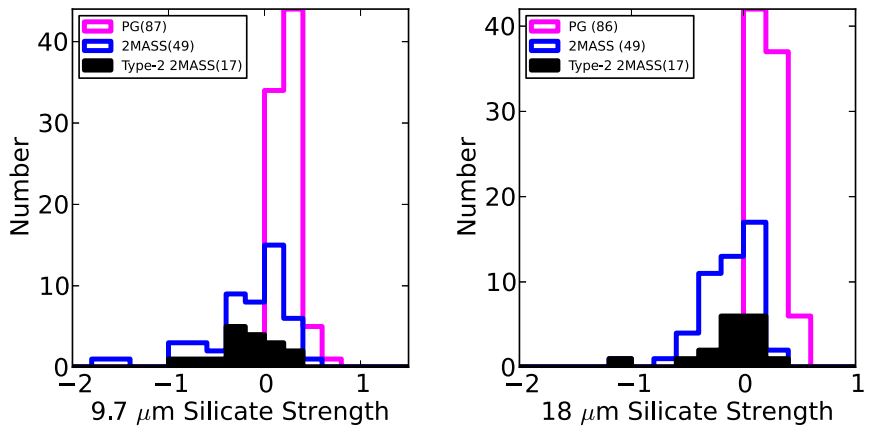

Figure 7. Distributions of silicate feature strengths for PG and 2MASS quasars. The $9.7 \mu \mathrm{m}$ feature is shown in the left panel and the $18 \mu \mathrm{m}$ feature is in the right panel.

(A color version of this figure is available in the online journal.)

tions are not always observed in other samples of galaxies and AGNs (Wu et al. 2010; Stierwalt et al. 2013). Wu et al. (2010) investigated the IR SEDs of about 300 galaxies and AGN selected to be brighter than $5 \mathrm{mJy}$ at $24 \mu \mathrm{m}$. They found their objects are divided into two categories, one with low $\mathrm{EW}_{6.2 \mu \mathrm{mPAH}}$ and $\log \left(f_{70 \mu \mathrm{m}} / f_{24 \mu \mathrm{m}}\right)$, and another with high $\mathrm{EW}_{6.2 \mu \mathrm{mPAH}}$ and $\log \left(f_{70 \mu \mathrm{m}} / f_{24 \mu \mathrm{m}}\right)$ but with no apparent correlation between $\mathrm{EW}_{6.2 \mu \mathrm{mPAH}}$ and $\log \left(f_{70 \mu \mathrm{m}} / f_{24 \mu \mathrm{m}}\right)$ within the category. Stierwalt et al. (2013) mainly focused on the IR SEDs of LIRGs and did not see a relationship between $f_{30 \mu \mathrm{m}} / f_{15 \mu \mathrm{m}}$ and $\mathrm{EW}_{6.2 \mu \mathrm{mPAH}}$ either. As mentioned by Stierwalt et al. (2013), the IR slope is not only affected by the star-forming contribution but is also affected by the obscuration, which could cause a steep IR spectrum even at low $\mathrm{EW}_{\mathrm{PAH}}$ if the energy source is heavily buried. Our quasar samples lack heavily buried sources, as indicated by the distributions of the silicate feature in Figure 7. The mid-IR slope is then mainly driven by the star-forming contribution and thus shows a correlation with the $\mathrm{EW}_{\mathrm{PAH}}$.

We further investigated the star-forming contribution at individual wavelengths ( 25 or $60 \mu \mathrm{m}$ ) and their trend with the $\mathrm{EW}_{\mathrm{PAH}}$. As shown in the middle panel of Figure 6 , the starforming fraction at rest-frame $25 \mu \mathrm{m}$ is usually below $30 \%$, indicating the emission at this wavelength is generally dominated by the radiation from the dusty torus. This fraction is correlated with the $\mathrm{EW}_{11.3 \mu \mathrm{mPAH}}$, as might be expected because the SF is not the dominant contributor, and both the quasar and star-forming SEDs do not change significantly from source to source. On the other hand, as shown in the right panel of Figure 6 , the rest-frame $60 \mu \mathrm{m}$ radiation is dominated by $\mathrm{SF}$,
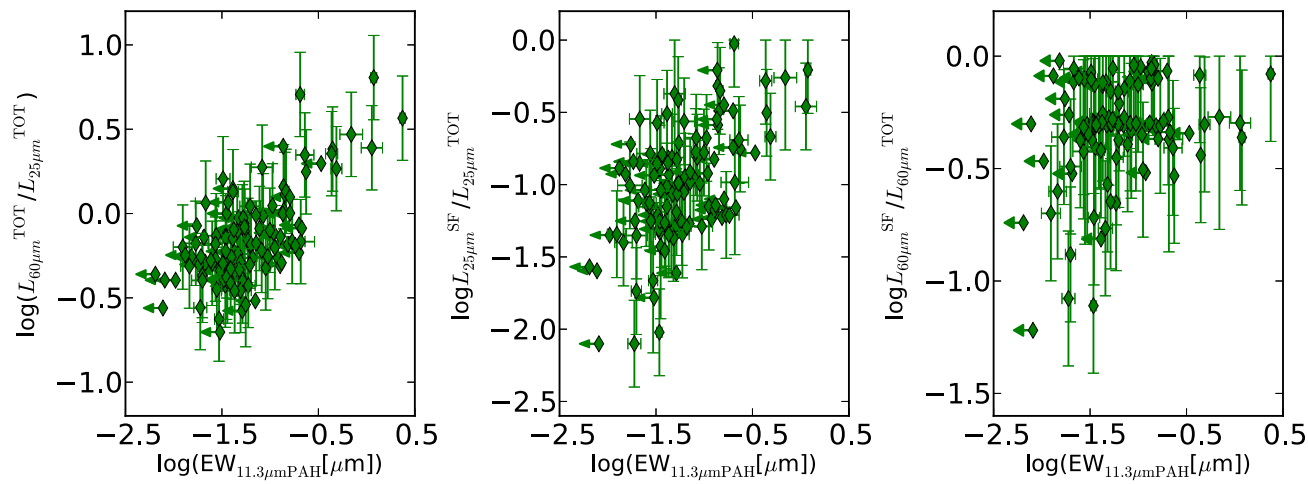

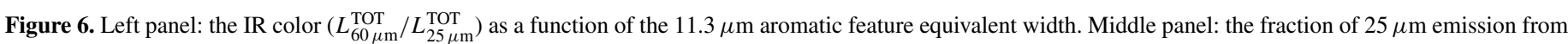

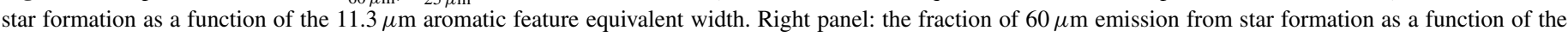
$11.3 \mu \mathrm{m}$ aromatic feature equivalent width.

(A color version of this figure is available in the online journal.) 


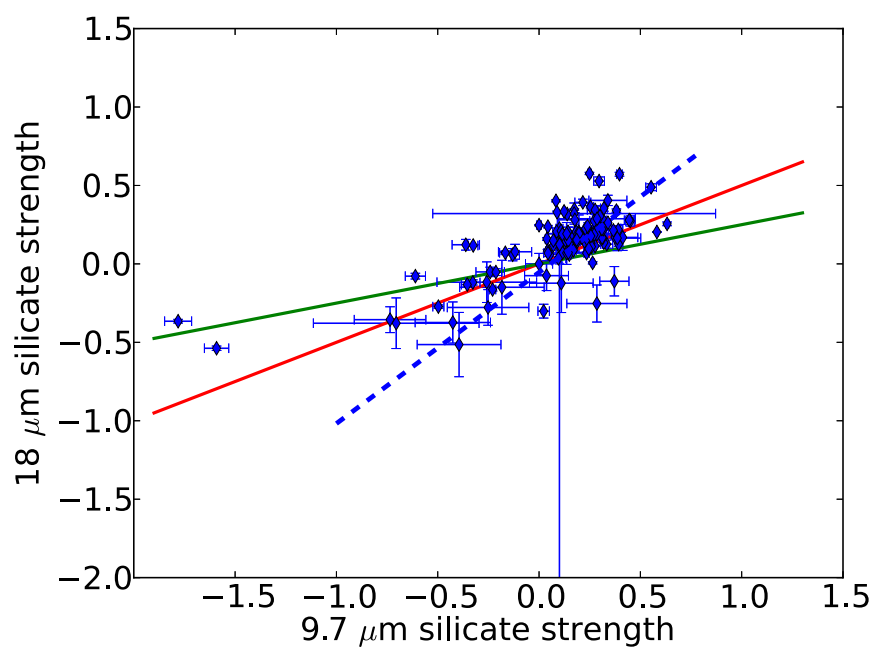

Figure 8. $18 \mu \mathrm{m}$ silicate feature strength vs. $9.7 \mu \mathrm{m}$ feature strength. Symbols indicate the measurements of PG and 2MASS objects where the dashed line is the best linear fit. The red and green areas are the predictions for clumpy and smooth dusty tori, respectively (Feltre et al. 2012).

(A color version of this figure is available in the online journal.)

with the star-forming fraction mostly above $30 \%$. This fraction does not show an apparent relation with the $\mathrm{EW}_{11.3 \mu \mathrm{mPAH}}$, again as might be expected since the two indicators are mainly affected by different processes, SF dominating the $60 \mu \mathrm{m}$ while $\mathrm{EW}_{11.3 \mu \mathrm{mPAH}}$ is a measure of the fraction of the output at this wavelength due to SF to that from nuclear accretion, since the latter dominates the $11.3 \mu \mathrm{m}$ continuum.

\subsection{Silicate Features}

Figure 7 shows the distributions of the 9.7 and $18 \mu \mathrm{m}$ silicate feature strengths including non-detections. The non-detected features are weak with $3 \sigma$ upper limits for $\ln \left(f^{\text {peak }} / f_{\text {cont }}^{\text {peak }}\right)$ between -0.3 and 0.3. As shown in the figure, PG quasars mainly show emission features while the majority of 2MASS quasars have features in absorption, which is consistent with the expectations from the unified model where the dusty tori of the unobscured quasars are viewed face-on and those of obscured quasars are seen edge-on. However, there is no good relation between the silicate feature and quasar optical type; e.g., many type 2 quasars show silicate features in emission. As indicated in the figure, the feature strength is moderate, with the majority in the range of $\ln \left(f^{\text {peak }} / f_{\text {cont }}^{\text {peak }}\right)=-0.5$ to 0.5 . The lack of strong $(>0.5$ or $<-0.5$ ) $9.7 \mu \mathrm{m}$ silicate emission features is consistent with expectations if the dusty torus is clumpy instead of smooth (Fritz et al. 2006; Hönig et al. 2006; Nenkova et al. 2008; Schartmann et al. 2008; Stalevski et al. 2012; Feltre et al. 2012).

Figure 8 shows the relationship between two silicate feature strengths for sources with $\mathrm{EW}_{11.3 \mu \mathrm{mPAH}}<0.2$. The two features are roughly correlated, with a Pearson value of 0.6 . A regression fit to the data points gives $S_{18 \mu \mathrm{m}}=0.96 S_{9.7 \mu \mathrm{m}}-0.05$ and $1 \sigma$ scatter of 0.83 . The relatively large scatter partly arises from the uncertainties in determining the underlying continuum. As shown in Figure 7, the small dynamic range of the two feature strengths will also limit the accuracy in the slope measurement. In Figure 8, we overlay some predictions of clumpy and smooth models (Feltre et al. 2012). The clumpy model produces a slope closer to the observations than the smooth model. In a dust geometry composed of clumps, the sides of individual clouds

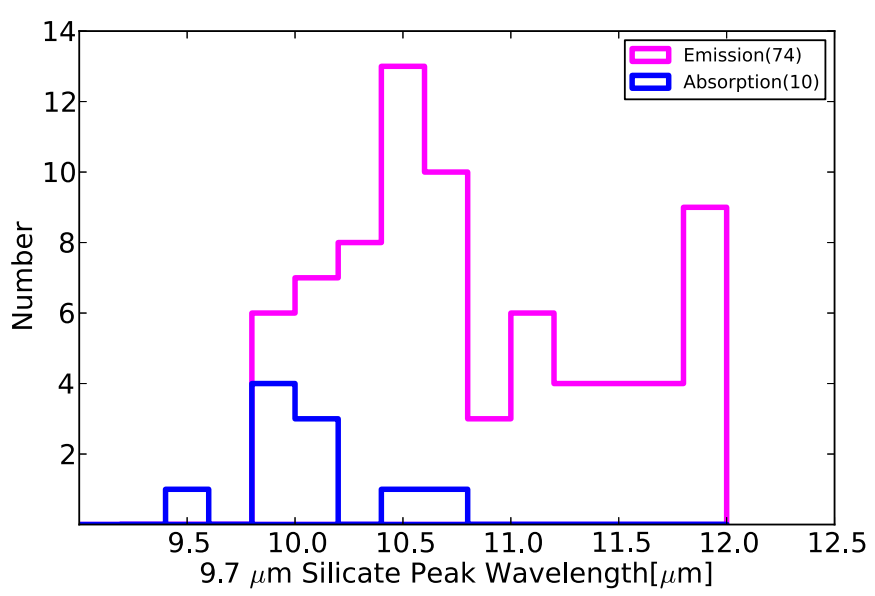

Figure 9. Distribution of the peak wavelength of the "9.7 $\mu \mathrm{m}$ " silicate feature for all cases with $\mathrm{S} / \mathrm{N}>5$.

(A color version of this figure is available in the online journal.)

facing the central nuclei are heated and produce silicate emission features while the absorption features are imposed on the output from the dark sides. In an inclined viewing angle toward the AGN, the foreground clouds partially block the view of the background ones. Therefore, a majority of clouds are viewed from the dark sides and silicate absorption features dominate, although some fraction of clouds are seen from their bright sides and the associated silicate emission reduces the strength of the absorption trough. As the $9.7 \mu \mathrm{m}$ feature has a larger optical depth than the $18 \mu \mathrm{m}$ one, implying a stronger emission feature at $9.7 \mu \mathrm{m}$ from the bright side of the cloud, the $9.7 \mu \mathrm{m}$ absorption feature is reduced more so that a steeper slope is produced.

Figure 9 shows the distribution of the peak wavelengths of $9.7 \mu \mathrm{m}$ features with signal-to-noise ratio $(\mathrm{S} / \mathrm{N})>5$. Almost all " $9.7 \mu \mathrm{m}$ " features peak in emission at wavelengths longer than $9.7 \mu \mathrm{m}$, the peak location of silicate features observed in Galactic sources or star-forming galaxies (Smith et al. 2010). Some of them even peak at the longest wavelength $(12 \mu \mathrm{m})$ that is allowed by our fit, indicating that they could peak at even longer wavelength. In contrast, those in absorption show minima at the expected $9.7 \mu \mathrm{m}$ wavelength. The offsets in the peak wavelengths of emission features in quasars have been observed previously in small samples (Siebenmorgen et al. 2005; Hao et al. 2005; Sturm et al. 2005, 2006; Shi et al. 2006). None of the existing dusty torus models that adopt Milky Way ISM dust composition predict such offsets (Fritz et al. 2006; Hönig et al. 2006; Nenkova et al. 2008; Schartmann et al. 2008; Stalevski et al. 2012; Feltre et al. 2012). We have used the library of embedded young stellar object SEDs from Whitney (2014) to test whether the offset could arise from radiative transfer effects, but find that our fitting procedure generally centers the silicate features near $9.7 \mu \mathrm{m}$ for them. We conclude that the offset is intrinsic to the quasars. Such differences between silicate absorption and emission features likely indicate that the physical properties of the emitting dust grains are subject to modifications due to exposure to the strong nuclear radiation, while "normal" dust grains in the host galaxies or outer cold edges of the dusty tori are responsible for the absorption features. It has been shown that for individual cases, the silicate emission feature in quasars can be well reproduced by adopting large size dust grains or modifying the dust compositions (Molster \& Waters 2003; Smith et al. 2010). 


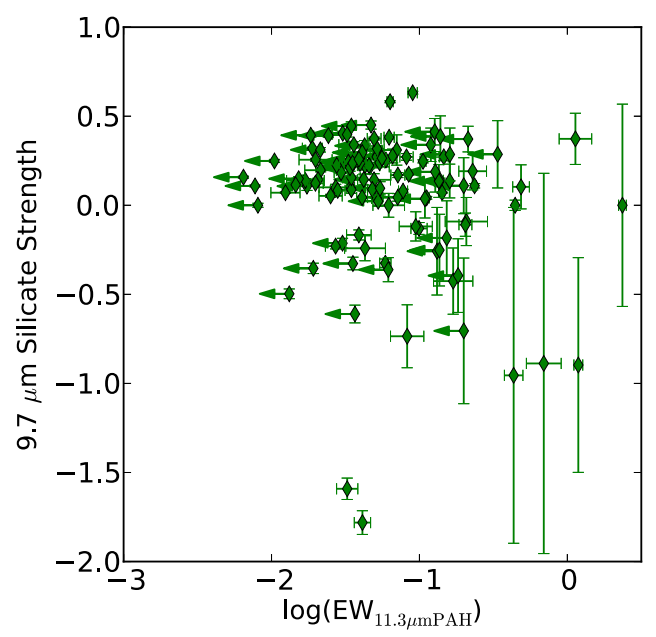

Figure 10. The $9.7 \mu \mathrm{m}$ silicate feature strength as a function of the $11.3 \mu \mathrm{m}$ aromatic feature equivalent width.

(A color version of this figure is available in the online journal.)

Figure 10 shows the $9.7 \mu \mathrm{m}$ silicate feature strength versus the EW of the $11.3 \mu \mathrm{m}$ aromatic feature. No relationship between the two is observed, which is also seen in other AGN samples (Hernán-Caballero et al. 2011). Unlike LIRGs and ULIRGs (Spoon et al. 2007; Stierwalt et al. 2013), our quasar sample does not show any deep silicate features (strength $<-1.5$ ) at any $\mathrm{EW}_{11.3 \mu \mathrm{mPAH}}$, while LIRGs and ULIRGs contain a branch in this plane that shows deeper silicate features at decreasing $\mathrm{EW}_{11.3 \mu \mathrm{mPAH}}$, pointing to a population of deeply buried sources.

\section{CONCLUSIONS}

We report mid-IR spectroscopy and mid- and far-IR photometry of the PG and 2MASS samples of quasars. We analyze these data by fitting for spectral features due to aromatics and silicates. The broad-band IR SEDs of both quasar types are similar. However, the PG quasars tend to have silicates in emission and the 2MASS ones have them in absorption. We show that the SFRs estimated from the aromatic features and the far-IR luminosities of these quasars are consistent. Emission due to SF in the host galaxies dominates the outputs of these systems at $160 \mu \mathrm{m}$, but at $70 \mu \mathrm{m}$ and low SFRs it is likely that the quasar contaminates the star-forming SED significantly. The peak wavelength of the " $10 \mu \mathrm{m}$ " silicate feature tends to be longer than observed in the general ISM, suggesting that the grain properties are modified by proximity to the AGNs.

We thank the anonymous referee for helpful comments that improved the paper significantly. Y.S. acknowledges support for this work from Natural Science Foundation of China under the grant 11373021 and by the Strategic Priority Research Program "The Emergence of Cosmological Structures" of the Chinese Academy of Sciences, grant No. XDB09000000.

\section{REFERENCES}

Antonucci, R. 1993, ARA\&A, 31, 473

Balog, Z., Müller, T., Nielbock, M., et al. 2013, ExA, 37, 129

Boroson, T. A., \& Green, R. F. 1992, ApJS, 80, 109

Calzetti, D., Kennicutt, R. C., Engelbracht, C. W., et al. 2007, ApJ, 666, 870

Cutri, R. M., Nelson, B. O., Kirkpatrick, J. D., Huchra, J. P., \& Smith, P. S. 2001, in ASP Conf. Ser. 232, The New Era of Wide Field Astronomy, ed. R. Clowes, A. Adamson, \& G. Bromage (San Francisco, CA: ASP), 78

Diamond-Stanic, A. M., \& Rieke, G. H. 2010, ApJ, 724, 140

Diamond-Stanic, A. M., Rieke, G. H., \& Rigby, J. R. 2009, ApJ, 698, 623

Elvis, M., Wilkes, B. J., McDowell, J. C., et al. 1994, ApJS, 95, 1

Esquej, P., Alonso-Herrero, A., González-Martín, O., et al. 2014, ApJ, 780, 86 Feltre, A., Hatziminaoglou, E., Fritz, J., \& Franceschini, A. 2012, MNRAS, 426,120

Ferrarese, L., \& Merritt, D. 2000, ApJL, 539, L9

Fritz, J., Franceschini, A., \& Hatziminaoglou, E. 2006, MNRAS, 366, 767

Gebhardt, K., Bender, R., Gary, B., et al. 2000, ApJL, 539, L13

Gordon, K. D., Rieke, G. H., Engelbracht, C. W., et al. 2005, PASP, 117,503

Goldschmidt, P., Miller, L., La Franca, F., \& Cristiani, S. 1992, MNRAS, 256,65

Hao, L., Spoon, H. W. W., Sloan, G. C., et al. 2005, ApJL, 625, L75

Hernán-Caballero, A., \& Hatziminaoglou, E. 2011, MNRAS, 414, 500

Hernán-Caballero, A., Pérez-Fournon, I., Hatziminaoglou, E., et al. 2009, MNRAS, 395, 1695

Hiner, K. D., Canalizo, G., Lacy, M., et al. 2009, ApJ, 706, 508

Hönig, S. F., Beckert, T., Ohnaka, K., \& Weigelt, G. 2006, A\&A, 452, 459

Jaffe, W., Meisenheimer, K., Röttgering, H. J. A., et al. 2004, Natur, 429, 47

Jester, S., Schneider, D. P., Richards, G. T., et al. 2005, AJ, 130, 873

Kellermann, K. I., Sramek, R., Schmidt, M., Shaffer, D. B., \& Green, R. 1989, AJ, 98, 1195

Kormendy, J., \& Richstone, D. 1995, ARA\&A, 33, 581

Lutz, D., Sturm, E., Tacconi, L. J., et al. 2008, ApJ, 684, 853

Magorrian, J., Tremaine, S., Richstone, D., et al. 1998, AJ, 115, 2285

Molster, F. J., \& Waters, L. B. F. M. 2003, in Astromineralogy, ed. Th. Henning (Lecture Notes in Physics, Vol. 609; Berlin: Springer), 121

Nenkova, M., Sirocky, M. M., Nikutta, R., Ivezić, Ž., \& Elitzur, M. 2008, ApJ, 685,160

Netzer, H., Lutz, D., Schweitzer, M., et al. 2007, ApJ, 666, 806

Peeters, E., Spoon, H. W. W., \& Tielens, A. G. G. M. 2004, ApJ, 613, 986

Richards, G. T., Lacy, M., Storrie-Lombardi, L. J., et al. 2006, ApJS, 166, 470

Rieke, G. H., Alonso-Herrero, A., Weiner, B. J., et al. 2009, ApJ, 692, 556

Rosario, D. J., Trakhtenbrot, B., Lutz, D., et al. 2013, A\&A, 560, A72

Schartmann, M., Meisenheimer, K., Camenzind, M., et al. 2008, A\&A, 482,67

Schmidt, M., \& Green, R. F. 1983, ApJ, 269, 352

Shang, Z., Brotherton, M. S., Wills, B. J., et al. 2011, ApJS, 196, 2

Shi, Y., Ogle, P., Rieke, G. H., et al. 2007, ApJ, 669, 841

Shi, Y., Rieke, G. H., Hines, D. C., et al. 2006, ApJ, 653, 127

Siebenmorgen, R., Haas, M., Krügel, E., \& Schulz, B. 2005, A\&A, 436, L5

Smith, H. A., Li, A., Li, M. P., et al. 2010, ApJ, 716, 490

Smith, J. D. T., Draine, B. T., Dale, D. A., et al. 2007, ApJ, 656, 770

Smith, P. S., Schmidt, G. D., Hines, D. C., Cutri, R. M., \& Nelson, B. O. 2002, ApJ, 569, 23

Spoon, H. W. W., Marshall, J. A., Houck, J. R., et al. 2007, ApJL, 654, L49

Stalevski, M., Fritz, J., Baes, M., Nakos, T., \& Popović, L. Č. 2012, MNRAS, 420, 2756

Stierwalt, S., Armus, L., Surace, J. A., et al. 2013, ApJS, 206, 1

Sturm, E., Hasinger, G., Lehmann, I., et al. 2006, ApJ, 642, 81

Sturm, E., Schweitzer, M., Lutz, D., et al. 2005, ApJL, 629, L21

Vega, O., Bressan, A., Panuzzo, P., et al. 2010, ApJ, 721, 1090

Veilleux, S., Rupke, D. S. N., Kim, D.-C., et al. 2009, ApJS, 182, 628

Whitney, B. 2014, http://caravan.astro.wisc.edu/protostars/

Wu, Y., Helou, G., Armus, L., et al. 2010, ApJ, 723, 895 